\title{
PEGylated liposomes associate with Wnt3A protein and expand putative stem cells in human bone marrow populations
}

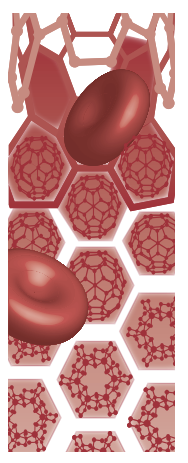

\begin{abstract}
Aim: To fabricate PEGylated liposomes which preserve the activity of hydrophobic Wnt3A protein, and to demonstrate their efficacy in promoting expansion of osteoprogenitors from human bone marrow. Methods: PEGylated liposomes composed of several synthetic lipids were tested for their ability to preserve Wnt3A activity in reporter and differentiation assays. Single-molecule microspectroscopy was used to test for direct association of protein with liposomes. Results: Labeled Wnt3A protein directly associated with all tested liposome preparations. However, Wnt3A activity was preserved or enhanced in PEGylated 1,2-dioleoyl-sn-glycero-3phosphocholine (DOPC) liposomes but not in PEGylated 1,2-dimyristoyl-sn-glycero3-phosphocholine (DMPC) liposomes. PEGylated Wnt3A liposomes associated with skeletal stem cell populations in human bone marrow and promoted osteogenesis. Conclusion: Active Wnt protein-containing PEGylated liposomes may have utility for systemic administration for bone repair.
\end{abstract}

First draft submitted: 3 November 2016; Accepted for publication: 7 February 2017; Published online: 29 March 2017

Keywords: fracture repair $\bullet$ liposomes $\bullet$ marrow stromal cells $\bullet$ mesenchymal stem cells - osteoprogenitors $\bullet$ PEGylated liposomes $\bullet$ regenerative medicine $\bullet$ skeletal stem cells $\bullet$ Wnt

Liposomes are artificial vesicles, ranging in size from approximately $25 \mathrm{~nm}$ to $2.5 \mu \mathrm{m}$, which may be composed of a variety of synthetic or naturally derived phospholipids [1]. They have found wide use in both basic science and in the pharmaceutical industry, and more than 15 liposome-based drugs currently have clinical approval for a range of medical applications, including infection control [2], vaccine delivery [3] or anticancer medications [4]. Many more are in current clinical trials [5]. They are beneficial for drug delivery when they are able to offer superior therapeutic efficacy and/or safety over existing formulations. This is based on several important advantages, including improved solubility, protection of an entrapped drug from degradation, increased cellular uptake and improvements in a drug's pharmacokinetic and tissue distribution profile. A major step forward for clinical applications came with the advent of 'stealth' liposomes, which display PEG on their surfaces ('PEGylated' liposomes) [6]. PEGylated liposomes exhibit prolonged circulation times as they are better able to evade uptake and removal by the reticular endothelial system. Long-circulating nano-sized ( $100 \mathrm{~nm}$ diameter) PEGylated liposomes preferentially accumulate and concentrate drugs within solid tumors due to the 'enhanced permeability and retention' effect which, while not entirely understood, is thought to be a result from an immature and leaky vasculature within the tumor [7,8].

While the primary success of circulating liposomal therapies has been in the field of cancer sciences, evidence is emerging that liposomes may be attractive drug delivery vehicles for other indications, including regenerative medicine [9]. Liposomes can
Agnieszka A Janeczek1, Edoardo Scarpa', Mathew H Horrocks ${ }^{4}$, Rahul S Tare', Caroline A Rowland ${ }^{3}$, Dominic Jenner ${ }^{3}$, Tracey A Newman'2, Richard OC Oreffo', Steven F Lee ${ }^{4}$ \& Nicholas D Evans ${ }^{*, 1}$

${ }^{1}$ Human Development \& Health Academic Unit, Faculty of Medicine, Centre for Human Development, Stem Cells \& Regeneration, Bone \& Joint Research Group \& Institute for Life Sciences, Institute for Developmental Sciences, University of Southampton, Tremona Road, Southampton, SO16 6YD, UK

${ }^{2}$ Clinical \& Experimental Sciences, Faculty of Medicine, Institute for Life Sciences, University of Southampton, Building 85, Life Sciences Building, Highfield Campus, Southampton, S017 1BJ, UK

${ }^{3}$ Microbiology Group, Chemical, Biological \& Radiological Division, Dstl, Porton Down, Salisbury, SP4 OJQ, UK ${ }^{4}$ Department of Chemistry, University of Cambridge, Cambridge, CB2 1EW, UK

*Author for correspondence:

Tel.: +4402381203293

n.d.evans@soton.ac.uk 
carry a wide range of cargoes, including small molecules, nucleic acids, proteins and peptides, and therefore may act as systemic or local delivery systems for therapeutic agents that might otherwise be ineffective due to poor bioavailability or due to degradation. For example, liposomes have been used to deliver a variety of molecules when tethered to biomaterial scaffolds in order to promote regeneration [10], or have been used systemically to entrap and deliver poorly soluble molecules such as dexamethasone [11], or highly soluble molecules such as bisphosphonates [12,13]. Added functionality may be achieved by including surface modification to target liposomes to a desired tissue. For example, liposomes have been modified with surface groups including (AspSerSer) ${ }_{6}$ or bisphosphonate [14,15], type II collagen antibody [16] or E-selectin aptamer [17] for targeting to bone, cartilage and bone marrow, respectively.

We are investigating the notion that liposomes can be used for the systemic delivery of Wnt proteins. Wnt proteins are a family of secreted glycoprotein growth factors which, on binding to cell surface receptors Frizzled and Lrp5/6, activate the intracellular Wnt signaling pathway. Activation of Wnt signaling may have a diverse range of effects and is highly dependent on the target cell or tissue, but is particularly important in bone homeostasis and disease $[18,19]$. Several humanized monoclonal antibodies which bind to and inactivate inhibitors of Wnt signaling, such as DKK1 [20] or SOST [21] are currently in clinical trials, with recent data suggesting efficacy in promoting bone formation in osteoporotic patients [22,23]. The efficacy of these monoclonal antibodies in fracture healing is less clear, however, which may be related to their prolonged in vivo activity (with half-lives of $>4$ weeks [24]), and the fact that it is known that elevated Wnt signaling can have both positive and negative effects depending on the timing of delivery [25]. In these cases, delivery of short-acting molecules such as Wnt proteins rather than antibodies will provide much better control of temporal activation of signaling, as well as the potential of selective localization of the molecule to the injury site.

As Wnt proteins carry fatty acid modifications which render them unstable in aqueous solution [26,27], liposome incorporation (for one member of the Wnt family, Wnt3A) has been shown to be an effective method of preserving both in vitro and in vivo stability and activity [28-32]. However, several challenges and unanswered questions remain. Inclusion of PEGylated lipids has been shown to abolish the activity of current liposome preparations [30], restricting their use to local application or injection [29,32]. Local injection may disturb the hematoma or fracture callus (the integrity of which is important in healing) and in many cases may be difficult (requiring radiological expertise), painful and increase the risk of infection, therefore in many cases systemic delivery may be advantageous. It also remains unclear whether PEGylation prevents liposomal interaction with Wnt3A protein or inhibits subsequent cell interaction.

In this study, we focused on optimizing an active Wnt3A liposomal formulation incorporating PEGylation that could subsequently be used for systemic delivery. We tested the hypothesis that Wnt3A can stably associate with neutrally charged $100 \mathrm{~nm}$ 'stealth' liposomes, remain active in these particles and can be efficiently delivered to skeletal stem cells. We also provide, for the first time, evidence of a direct interaction of Wnt proteins with liposomal carriers by using single-molecule microspectroscopy techniques. Finally, we determined the optimal liposome composition and measured its relative uptake by different cell populations found in bone marrow isolates in vitro. Our results demonstrate that incorporation into PEGylated DOPC liposomes enhanced Wnt3A protein activity and that these liposomes can be used as delivery vesicles of Wnt3A to stromal/skeletal stem cell populations of the bone marrow. A schematic overview of our approach is presented in Figure 1.

\section{Materials \& methods}

For full methods, please refer to the supplementary information.

\section{Bone marrow cells}

Bone marrow mononuclear cells (BMMNCs) were isolated from femoral samples obtained from hematologically normal individuals undergoing hip replacement surgery at Southampton General Hospital or Spire Hospital Southampton, with the approval of the appropriate Local Research Ethics Committee (LREC 194/99/1), as previously described [33]. Cells were maintained in basal medium ( $\alpha$-MEM containing $10 \% \mathrm{FBS}$ and $100 \mu \mathrm{g} / \mathrm{ml}$ penicillin/streptomycin) or osteogenic medium (basal medium supplemented with $100 \mu \mathrm{M}$ ascorbate-2-phosphate, $10 \mathrm{nM}$ dexamethasone and $5 \mathrm{mM} \beta$-glycerophosphate), with the exception of when exposed to Wnt3A protein (5036-WN, R\&D Systems, McKinley, MN, USA) or liposomes, where serum concentration in the media was $5 \%$.

\section{Liposome preparation}

A total of $14 \mu$ moles of lipids (Avanti Polar Lipids, AL, USA) were dissolved in $1 \mathrm{ml}$ chloroform/methanol (3:1 $\mathrm{v} / \mathrm{v})$ in a $10-\mathrm{ml}$ round-bottomed flask, and dried to a thin film under a nitrogen gas stream for $5 \mathrm{~min}$, with further vacuum desiccation overnight at room tem- 


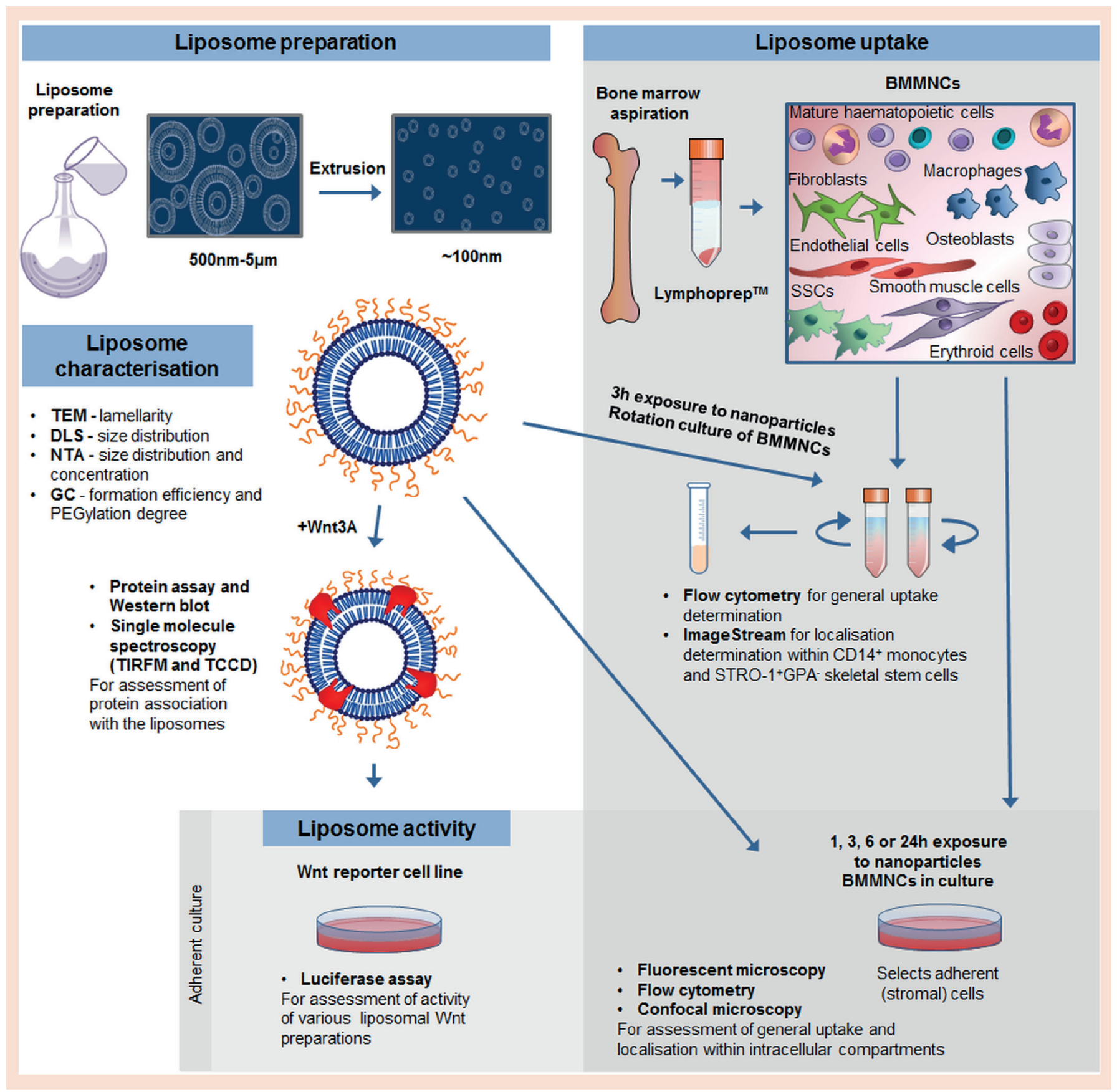

Figure 1. A schematic overview of the experimental approach of the study.

perature. Liposome PEGylation was achieved by adding 5 molar\% 1,2-distearoyl-sn-glycero-3-phosphoethanolamine-N-[amino(polyethylene glycol)] (DSPE-PEG) lipid to either DMPC or DOPC lipids. The thin lipid layer was then hydrated with $1 \mathrm{ml}$ phosphate-buffered saline (PBS), and placed in a water bath for $1 \mathrm{~h}$ at $37^{\circ} \mathrm{C}$ with vortexing every $10 \mathrm{~min}$. Extrusion was carried out on a heating block (Avanti) at $37^{\circ} \mathrm{C}, 41$-times through a $100 \mathrm{~nm}$ polycarbonate membrane (GE Healthcare/ Whatman, Little Chalfont, UK). In order to minimize the contamination of the liposome samples with free lipids, the solutions were ultracentrifuged (Beckman Coulter, CA, USA) at $100,000 \times g$ for $60 \mathrm{~min}$ at $4^{\circ} \mathrm{C}$. The liposome pellet was then resuspended in $1 \mathrm{ml}$ PBS and liposomes were subsequently stored at $4^{\circ} \mathrm{C}$. Loading of Wnt3A was carried out either during lipid film hydration, with $2 \mu \mathrm{g} / \mathrm{ml}$ of Wnt3A in PBS or after liposome formation, by incubation with $2 \mu \mathrm{g} / \mathrm{ml} \mathrm{Wnt3A}$ at room temperature, for up to $24 \mathrm{~h}$, followed by ultracentrifugation and resuspension in PBS at the initial volume. 


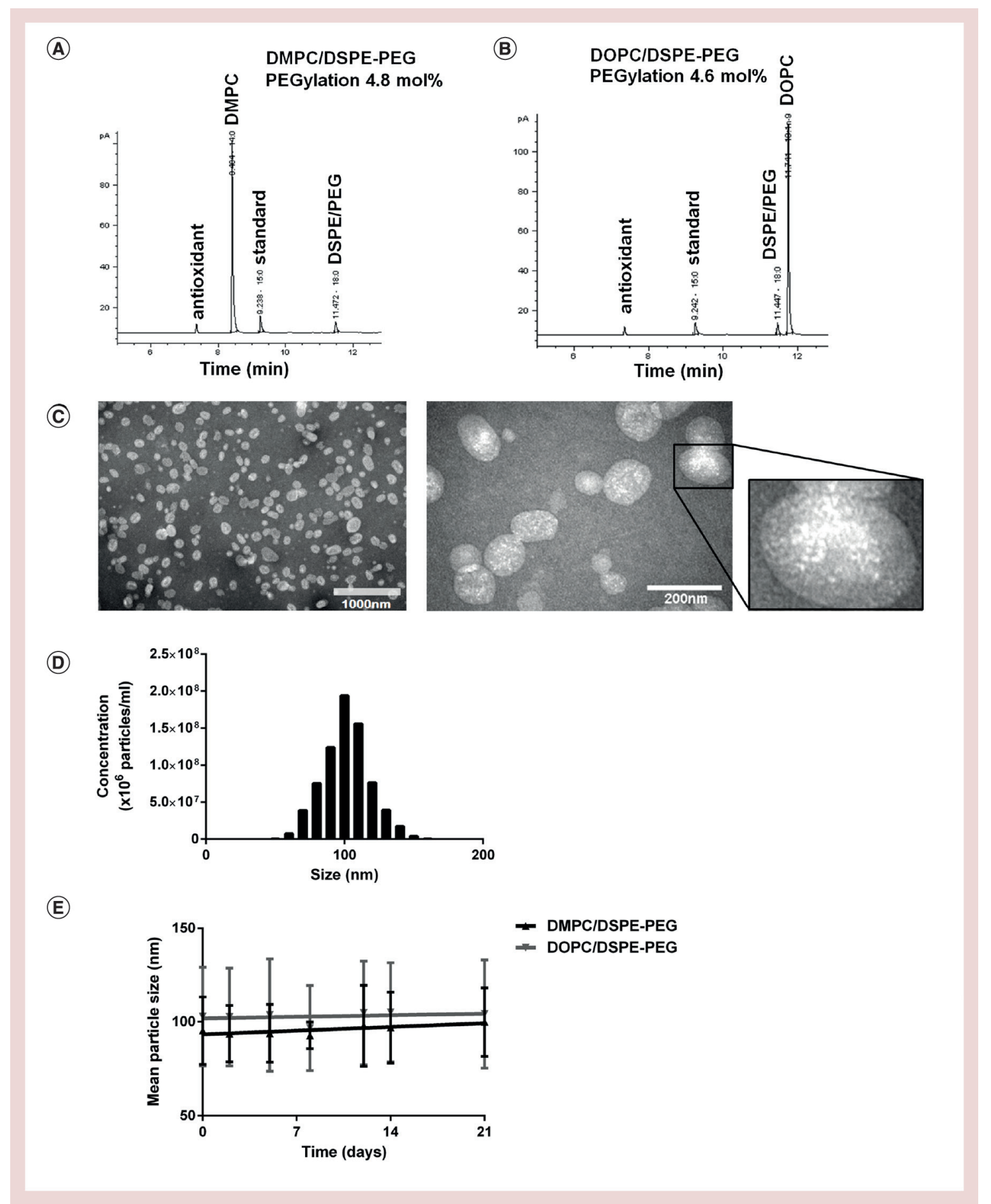

Figure 2. DMPC and DOPC PEGylated liposomes are stable, unilamellar nanoparticles of approximately $100 \mathrm{~nm}$ in size and low polydispersity. PEGylation degree quantified by GC reached approximately 5 mol\% as expected for both DMPC (A) and DOPC (B) liposomes. TEM micrographs of fixed and negatively stained liposome preparations (C), at 20,000x magnification showing uniform and unilamellar appearance of liposomes, and at 80,000x magnification showing uneven structures attributable to PEGylation and fluidity changes of the liposomal bilayer. Nanoparticle tracking analysis showed a narrow size distribution $(\sim 100 \mathrm{~nm})$ of liposomal preparations (D) and provided information on nanoparticle concentration $\left(10^{14}\right.$ particles $\left./ \mathrm{ml}\right)$. Size measurements by dynamic light scattering $(E ; n=3)$ confirmed size stability of the preparations for up to 21 days.

DMPC: 1,2-dimyristoyl-sn-glycero-3-phosphocholine; DOPC: 1,2-dioleoyl-sn-glycero-3-phosphocholine; GC: Gas chromatography. 
For preparation of fluorescent liposomes, $100 \mu \mathrm{M}$ of a lipophilic dye: $\mathrm{DiO}$ or DiI (Life Technologies, CA, USA) was mixed with the lipids before nitrogen drying. Where indicated, fluorescent liposomes were produced by incorporating a biotinylated lipid (DSPE-PEG Biotin, 2 molar\%; Avanti) into the lipid mixture and after formation incubated with streptavidin-AF647 conjugate (BioLegend, CA, USA) at a 1:1 biotin to streptavidin molar ratio for $20 \mathrm{~min}$ at room temperature.

\section{Liposomal Wnt activity assay}

The luciferase assay was performed on a 3T3 mouse embryonic fibroblast cell line (Enzo Life Sciences, NY, USA), modified to expresses the firefly luciferase reporter gene under the control of Wnt-responsive promoters TCF/LEF. Cells $\left(3 \times 10^{4}\right.$ cells/well, in $50 \mu \mathrm{l}$ assay media; 96-well plate) were treated with $100 \mathrm{ng} /$ $\mathrm{ml} \mathrm{Wnt3A} \mathrm{protein} \mathrm{or} 5 \mu \mathrm{l}$ liposomal Wnt3A in $50 \mu \mathrm{l}$ of DMEM. Plates were incubated at $37^{\circ} \mathrm{C}$ for $18 \mathrm{~h}$, followed by addition of $100 \mu \mathrm{l} /$ well of luciferase substrate diluted in luciferase buffer (Steady-Glo, Promega, WI, USA). After 10 min of reaction and cell lysis, the chemiluminescence signal was read $(0.1 \mathrm{~s} /$ well $)$ on a Varioscan Flash microplate reader (Thermo Fisher Scientific, MA, USA). To control for cell count, cell lysates were analyzed with the use of Quant-iT ${ }^{\text {TM }}$ PicoGreen ${ }^{\circledR}$ dsDNA Reagent (Thermo Fisher Scientific) according to manufacturer's protocol. Fluorescence was measured on an FLx800 fluorescence microplate reader (Biotek, VT, USA) with the excitation/emission wavelength set to $480 / 520 \mathrm{~nm}$, respectively.

\section{Protein quantification within the liposomal bilayer}

Quantification of Wnt3A protein in liposomal preparations was carried out using a fluorescent CBQCA Protein Quantitation Kit (Molecular Probes, OR, USA), according to the manufacturer's protocol.

\section{Single-molecule spectroscopy}

DiO-labeled liposomes and an Atto680-maleimidelabeled (Sigma, MO, USA) 'carrier-free' version of the Wnt3A protein (5036-WN/CF, R\&D Systems) as well as BSA (as negative control), or Alexa Fluor 647 (AF647)-streptavidin (Thermo Fisher Scientific; as positive control) were used for the single-molecule experiments. For TIRFM measurements, borosilicxate glass coverslips (VWR, PA, USA) were cleaned using an argon plasma cleaner (PDC-002, Harrick Plasma, NY, USA) for $1 \mathrm{~h}$ to remove any fluorescent residues and coated with $0.01 \%$ poly-L-lysine solution (Sigma). Next, $100 \mu \mathrm{l}$ of $100 \times$ diluted liposome sample was placed on each slide immediately prior to imaging, and $1 \mu \mathrm{l}$ of $4000 \times$ diluted $1 \mu \mathrm{M}$ protein was added to the slide. As a negative control, $0.1 \%$ of Triton-X in PBS was added to destroy the liposomes. TIRFM experiments were performed on a bespoke instrument [34] and were used to generate spatially resolved images (Figure 4E), this imaging technique axially restricts the detectable fluorescence signal to within approximately $200 \mathrm{~nm}$ from the sample glass/water interface of the coverslip. For each dataset, $3 \times 3$ image grids were measured in three different automated regions (Optiscan III, Prior, UK) to prevent user bias. Images were recorded at 33 frames per second for 100 frames, first from the red channel (Atto680 and AF647 emission) with $641 \mathrm{~nm}$ illumination, followed by 100 frames in the green channel (DiO emission) with $488 \mathrm{~nm}$ illumination. Singlemolecule confocal measurements were performed on a custom-built instrument described previously $[35,36]$. The experiments were performed in two color coincidence detection (TCCD) mode, in which light from 488 to $633 \mathrm{~nm}$ lasers were combined and focused to a diffraction limited spot, through which labeled molecules are able to diffuse, be excited and detected. For all confocal measurements, the samples were diluted to concentrations of approximately $100 \mathrm{pM}$, and the data were collected for $400 \mathrm{~s}$ (photons counted in timebins of $50 \mu \mathrm{s})$. As a negative control, $0.025 \%$ solution of 3-[(3-cholamidopropyl)dimethylammonio]-1-propanesulfonate (CHAPS) was added to destroy the liposomes. Data analysis was performed using customwritten procedures in Igor Pro (Wavemetrics, OR, USA). The co-ordinates of each detected spot in the green channel are analyzed in the red channel (after the frames are averaged over the whole stack and the background subtracted), and if there was a pixel above the applied red threshold value (mean intensity $+5 \mathrm{SD}$ ) then a positive coincidence is recorded. The number of chance coincident events was estimated by performing the previously described analyzes on green and red image stacks from different field-of-views. The association quotient (Q) for both methods was calculated according to the following equation:

$Q=\frac{C-E}{A-E}$

Where:

$\mathrm{Q}$ - association quotient;

$\mathrm{C}$ - number of coincident events in both red and green channels;

E - estimated rate at which coincident events occur by chance;

A - events rate in the green channel.

\section{Flow cytometry \& image stream}

Uptake of DiI-labeled liposomes by adherent bone marrow stromal cells (BMSCs) at P1 was studied by flow 
(A)

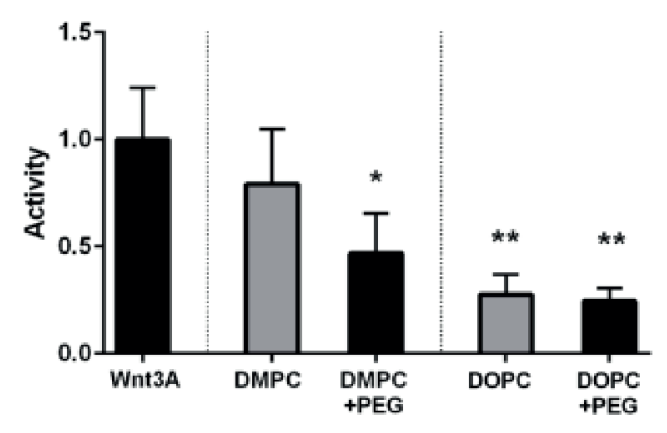

(B)

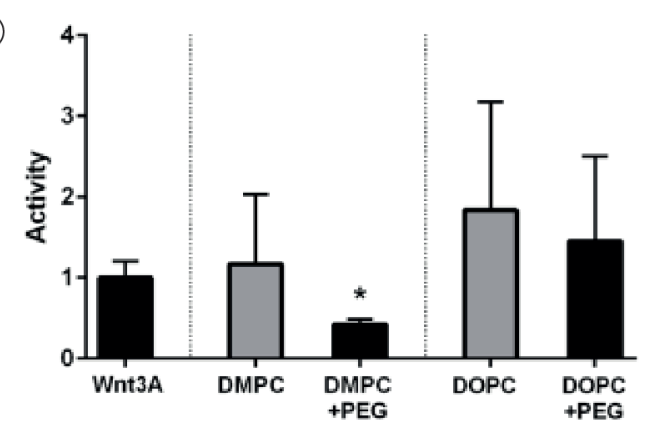

(C)

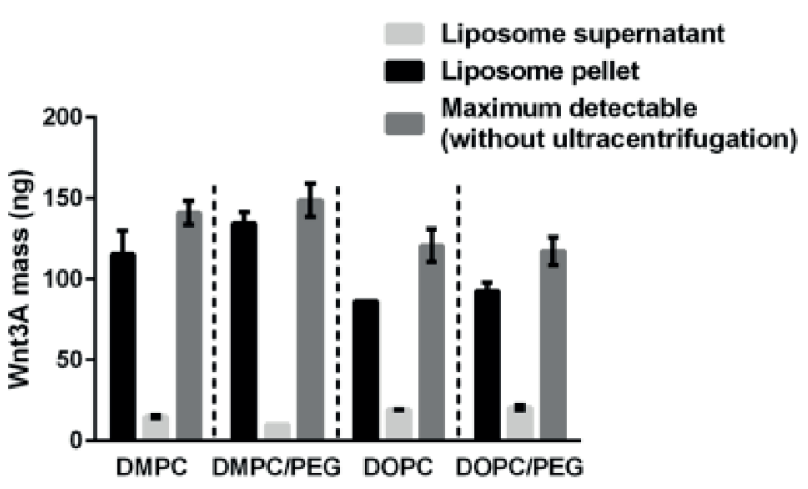

(D)

DMPC-based preparations

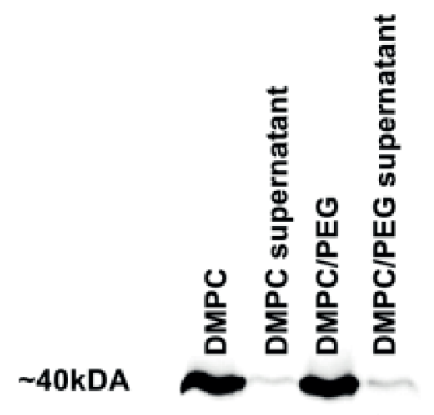

1.860 .362 .070 .41

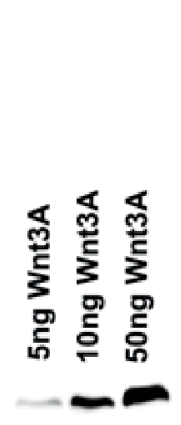

0.350 .571 .000
DOPC-based preparations

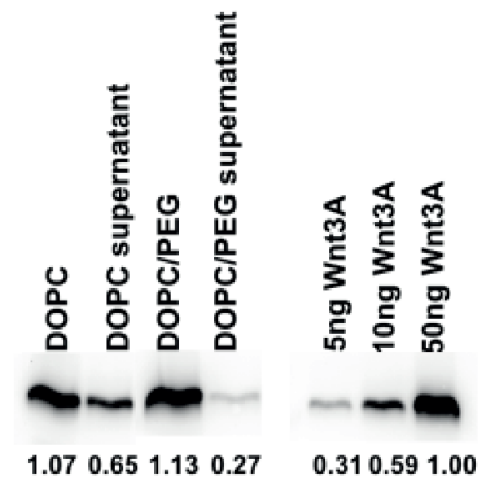

Figure 3. Wnt3A is active in PEGylated DOPC liposomes when associated passively post-extrusion. Addition of Wnt3A protein to lipid suspensions prior to extrusion resulted in loss of activity of all preparations except for DMPC (A), while incubation after formation (for $24 \mathrm{~h}$ ) resulted in liposome preparations with similar activities to native protein, except for DMPC + PEG (B). After Wnt3A liposome formation by incubation and subsequent ultracentrifugation, the majority of the protein remains in the liposomal pellet regardless of activity, as quantified by western blotting (C). This suggests that loss of activity in (A) is not due to loss of protein, but rather loss of protein activity. Efficiency of incorporation was calculated based on the Wnt3A mass associated with the liposomal pellet out of total detectable by assay and was: 90 and 79\% for DMPC/PEG and DOPC/PEG, respectively. Incubation for $24 \mathrm{~h}$ was sufficient to partition the majority of the Wnt3A protein into the liposome fraction (D), as confirmed by WB analysis of the liposomal pellets and supernatants. Data presented as mean $\pm S D, n=3$ separate experiments.

${ }^{*} p<0.05,{ }^{* *} p<0.01$ for liposome preparations versus Wnt3A reference. Statistical significance assessed by ANOVA with Tukey's correction.

DMPC: 1,2-dimyristoyl-sn-glycero-3-phosphocholine; DOPC: 1,2-dioleoyl-sn-glycero-3-phosphocholine. 
Table 1. Size and concentration of liposome preparations.

\begin{tabular}{|lll|}
\hline Preparation & DMPC/DSPE-PEG & DOPC/DSPE-PEG \\
\hline Mean (size) & $98.7 \mathrm{~nm}$ & $102 \mathrm{~nm}$ \\
\hline Mode (size) & $91.3 \mathrm{~nm}$ & $109.8 \mathrm{~nm}$ \\
\hline SD (size) & $25.4 \mathrm{~nm}$ & $23.1 \mathrm{~nm}$ \\
\hline d10 & $68.0 \mathrm{~nm}$ & $71.0 \mathrm{~nm}$ \\
\hline d50 & $90.9 \mathrm{~nm}$ & $96.4 \mathrm{~nm}$ \\
\hline d90 & $117.9 \mathrm{~nm}$ & $118.8 \mathrm{~nm}$ \\
\hline Concentration (particles/ml) & $1.44 \times 10^{14}$ & $1.66 \times 10^{14}$ \\
\hline PI & 0.084 & 0.051 \\
\hline $\begin{array}{l}\text { Summary of size, concentration and polydispersity index (PI) of the liposome preparations. d10, d50, d90 -\% levels below which the sample } \\
\text { population falls, giving an indication of the overall spread of size, in other words, 50\% of DMPC/PEG liposomes are } \leq 90.9 \mathrm{~nm} . \\
\text { DMPC: 1,2-dimyristoyl-sn-glycero-3-phosphocholine; DOPC: } 1,2-\text { dioleoyl-sn-glycero-3-phosphocholine; DSPE: 1,2-distearoyl-sn-glycero-3- } \\
\text { phosphoethanolamine. }\end{array}$ & \\
\hline
\end{tabular}

cytometry. Cells were seeded into 6 -well plates at $4 \times$ $10^{4}$ cells/well, allowed to adhere overnight and serum starved ( $\alpha$-MEM supplemented with $0.5 \%$ FBS and $100 \mathrm{U} / \mathrm{ml}$ penicillin and $100 \mu \mathrm{g} / \mathrm{ml}$ streptomycin per well) for $24 \mathrm{~h}$. Next, approximately $10^{7}$ liposomes/cell were added per well. After 1, 3, 6 and 24 h cells were trypsinized and assayed by flow cytometry on a Guava Easycyte Mini Flow Cytometer (Merck Millipore, MA, USA). Uptake of DiO-labeled or AF647-labeled liposomes was studied on fresh bone marrow isolates after $3 \mathrm{~h}$ of incubation in suspension with approximately $10^{6}$ liposomes/cell. Following this, immunostaining for CD14, STRO-1 and Glycophorin A (GPA) was carried out as described previously [25]. Cells were assayed on an FACS Canto II cytometer (BD Biosciences, NJ, USA) or Image Stream (Amnis, WA, USA). All flow cytometry experiments were analyzed using FlowJo v10 software. Data were acquired and analyzed as described previously [25].

\section{Statistical analysis}

Statistical analysis was performed using GraphPad Prism 6 software (GraphPad, CA, USA). Liposomal Wnt activity, uptake of liposomes and cell viability were analyzed using ANOVA with Tukey's post hoc correction. Curve fitting for liposome uptake was conducted using a hyperbola equation model. Data are presented as means \pm SD and the significance level was set at $\mathrm{p}<0.05$.

\section{Results}

PEGylated liposomes can be fabricated with uniform physical properties, \& are stable for prolonged time periods

We first fabricated liposomes by rehydration of lipids followed by extrusion through polycarbonate porous membranes. To make 'stealth' liposomes, which dis- play PEG groups on their surface, we incorporated $5 \%$ molar mass of DSPE-PEG prior to rehydration of DMPC or DOPC liposomes.

To confirm PEGylation, we measured liposome composition using gas chromatography (Figure 2A \& B). We found that DSPE-PEG was successfully incorporated in liposomes with the predicted efficiency (4.8 $\mathrm{mol} \%$ for DMPC and $4.6 \mathrm{~mol} \%$ for DOPC liposomes). Gas chromatograms of homogenous DMPC or DOPC liposomes did not show any peak for DSPE, as expected (Supplementary Figure 1A \& B).

We next analyzed size distribution, unilamellarity and stability of PEGylated liposomes. TEM imaging of dispersed PEGylated DMPC liposomes revealed uniform size distribution and no evidence of multilamellarity (Figure 2C). We also observed uneven structures with higher electron-absorbing properties in the liposomes, likely attributable to PEGylation due to fluidity changes of the liposomal bilayer (Figure 2C, inset). Nanoparticle tracking analysis, an imaging technique which measures the Brownian movement of nanoparticles by their light scattering properties, revealed a narrow size distribution of liposome preparations $(\sim 100 \mathrm{~nm})$, low polydispersity index and final nanoparticle concentrations of approximately $10^{14}$ particles $/ \mathrm{ml}$ (Figure 2D \& Table 1). Liposomes were stable in size for up to 21 days, with no change in the hydrodynamic radius as measured by dynamic light scattering (Figure 2E).

\section{Wnt3A protein is active in 'stealth' liposomal preparations}

Next, we tested the hypothesis that PEGylated liposomes can support the activity of incorporated Wnt protein, a necessary property for the use of liposomes as circulating Wnt carriers. A previous report [30] showed that inclusion of DSPE-PEG in otherwise active Wntcontaining liposome preparations abrogated the activ- 
(A)

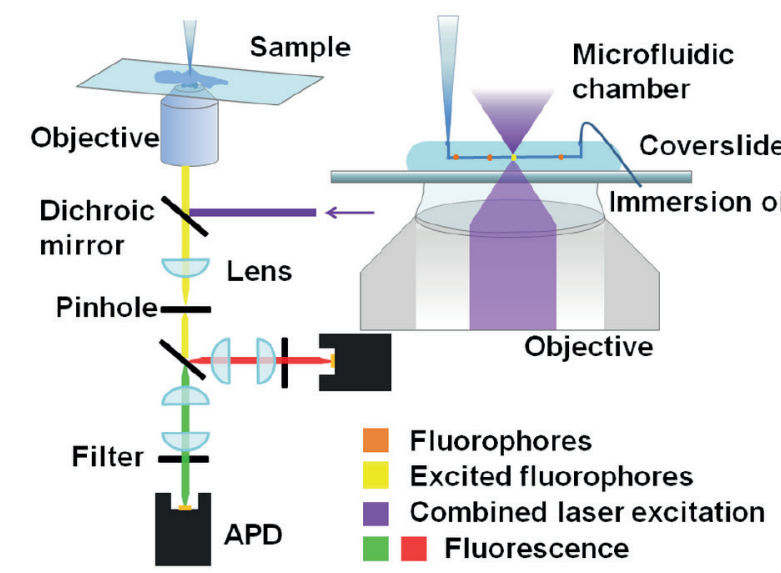

(D)

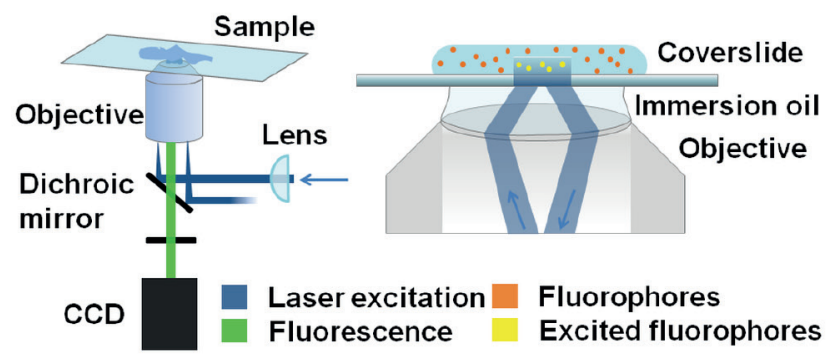

(B)
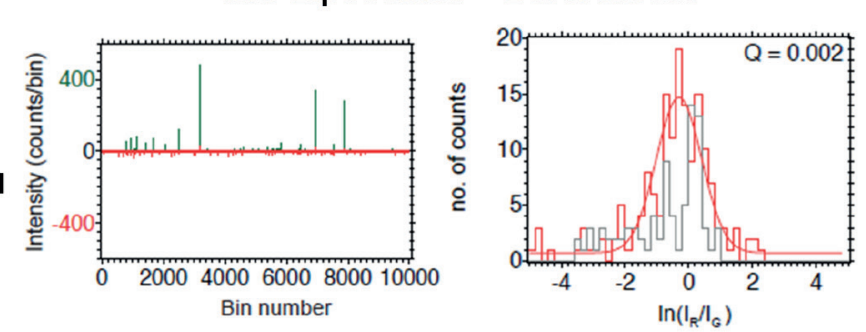

(C)

DiO Liposomes + Wnt3A Atto680
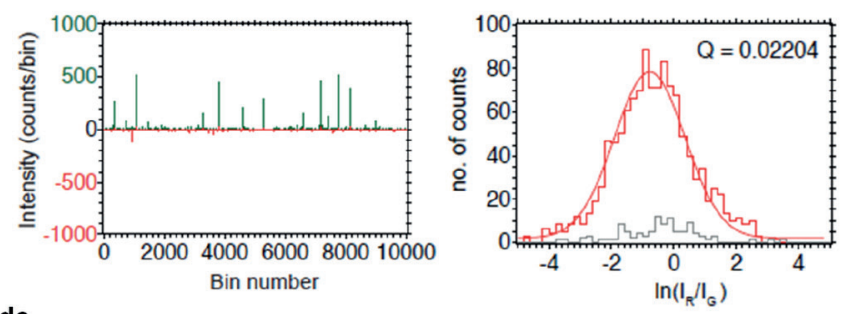

(E)
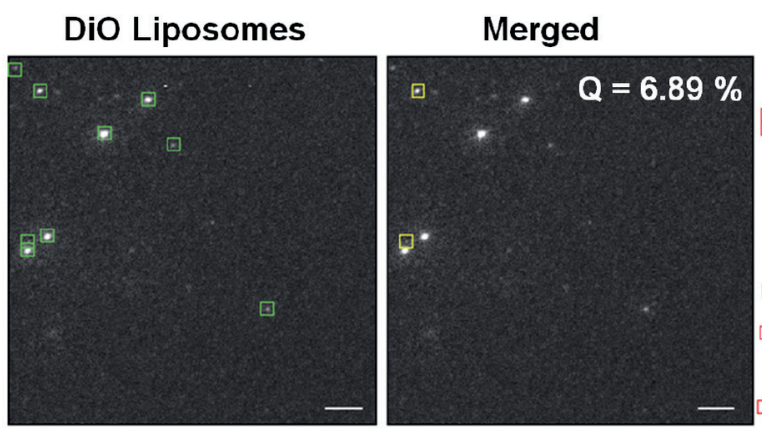

BSA Atto680

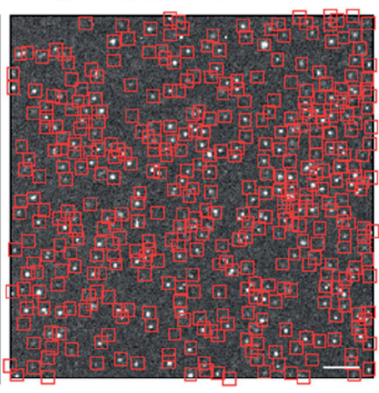

(F)
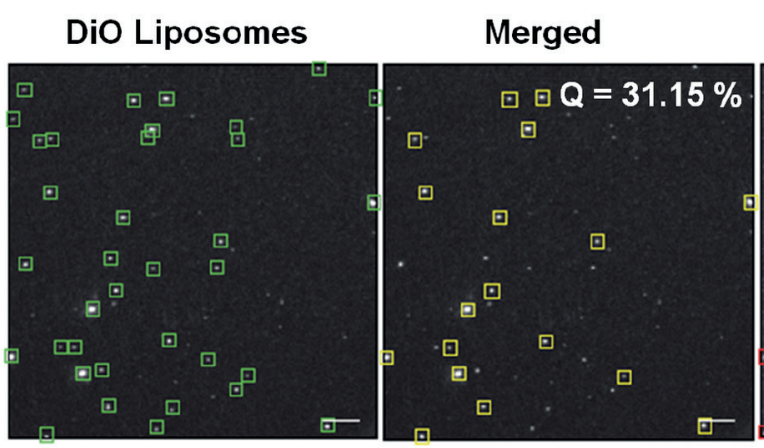

Wnt3A Atto680

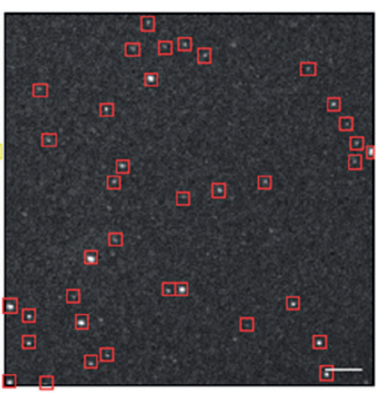


Figure 4. Wnt association with liposomes measured at the single-molecule level (see facig page). Wnt3A association with liposomes was confirmed by two single-molecule spectroscopy techniques, single-molecule confocal microscopy TIRFM, shown schematically in (A \& D), respectively. Using these techniques, association of labeled Wnt3A or control protein (BSA) with DiO-labeled liposomes was determined using the Q-value (see 'Materials \& methods' section). TCCD showed that even when working with picomolar concentrations of both liposomes and proteins in a microfluidic setup, the association of Wnt3A (C) with liposomes is $10 \times$ higher than that for BSA with liposomes ( $\mathrm{B} ; \mathrm{Q}=2.20$ vs $0.20 \%$, red lines on histograms). The gray lines show events that are due to multiple nonassociated molecules giving rise to coincidence due to their chance simultaneous occupation of the confocal volume (see 'Materials \& methods' section). Images recorded on TIRFM showed 31.15\% level of coincidence for Wnt3A protein (F) versus $6.89 \%$ for BSA (E), even when the control protein was tested at a higher concentration. Scale bars $=5 \mu \mathrm{m}$.

TCCD: Two-colour coincidence detection; TIRFM: Total internal reflection fluorescence microscopy.

ity of the protein. We considered that Wnt3A-liposome activity could be preserved by careful optimization of liposome composition and preparation. We examined two types of preparations, one where the protein was added prior to lipid extrusion, or another by incubation with formed liposomes post extrusion.

When Wnt3A was added prior to extrusion, the resultant DMPC liposomes had activities that were not significantly different from protein alone $(\mathrm{p}=0.36$; Figure $3 \mathrm{~A}$ ), assuming complete incorporation of protein with the liposomes. This may represent an underestimation of activity, as it is likely that some protein was lost to supernatant or precipitation and therefore (as previously reported) it is possible that DMPC liposome incorporation augments Wnt3A activity. PEGylation had a negative effect on liposome activity, resulting in a decrease in activity of DMPC liposomes $(58 \pm 6.3 \%$ of in the initial DMPC activity, $\mathrm{p}<0.05)$. DOPC-Wnt3A liposomes were less active than Wnt3A $(27 \pm 9 \%$ of Wnt3A reference activity, $\mathrm{p}<0.01$ ), but PEGylation had no effect on their activity.

In contrast, when Wnt3A was incorporated into liposomes after their formation, by incubation of the protein for $24 \mathrm{~h}$ in the presence of the liposomes, the activity of Wnt3A-DOPC liposomes was unchanged or increased compared with Wnt3A (Figure 3B). Moreover, PEGylation had no effect on the activity of DOPC liposomes $(p=0.65)$. In agreement with previous studies [28], we found that $24 \mathrm{~h}$ of incubation of Wnt3A with either PEGylated or unPEGylated liposomes was sufficient to partition the majority of the Wnt3A protein into the liposome fraction. $>80 \%$ of protein was found in the pellet fraction of ultracentrifuged liposome suspension at this time point, measured by a protein quantification assay and western blotting (Figure 3C \& D). Wnt3A incorporation efficiency varied between liposomal preparations (90 and $79 \%$ for DMPC/PEG and DOPC/PEG, respectively). This process of association of protein with liposomes was time-dependent, with an increasing mass of Wnt3A protein found in the pellet with longer incubation times (Supplementary Figure 2). The size and distribution of Wnt3A-associated DOPC liposomes was not significantly different from that of control liposomes (mean $112 \mathrm{~nm} \pm 27 \mathrm{~nm} \mathrm{SD}, \mathrm{n}=3$ ) .
Together these data demonstrate that PEGylated liposome preparations are suitable for maintaining the activity of Wnt $3 \mathrm{~A}$ and therefore can be used as delivery vesicles for this protein.

\section{Wnt3A association with liposomes can be visualized at the single-molecule level}

While the biochemical methods described above are useful for determining the association of a protein with a given fraction of a particulate suspension, they do not directly reveal spatial interactions of proteins with liposome carrier. To prove such interactions, we next employed single-molecule microscopic methods, two-colour coincidence detection (TCCD) and total internal reflection fluorescence microscopy (TIRFM). For visualization, liposomes were labeled with the lipophilic fluorescent dye, $\mathrm{DiO}$ and $\mathrm{Wnt} 3 \mathrm{~A}$ protein was labeled with an Atto680-maleimide, which reacts with sulphydryl groups on proteins. As a positive control, biotinylated liposomes incubated with AF647-labeled streptavidin were prepared. Atto680-labeled BSA was used as negative control, as BSA is not predicted to interact with membranes.

In single-molecule confocal TCCD measurements, 488 and $633 \mathrm{~nm}$ radiation are combined and focused to a diffraction-limited volume [36,37] (see Figure 4A for cartoon schematic). As molecules transit this probe volume, they may give rise to a green burst of fluorescence if tagged with a $488 \mathrm{~nm}$ excitable dye, and a red burst if tagged with a $633 \mathrm{~nm}$ excitable dye. If both types of dye are present, there will be a simultaneous 'coincident' burst in both channels. Measurements were first performed on a dual-labeled (AF488 and AF647) DNA duplex (Supplementary Figure 3A), to establish a value for the maximum measurable co-incidence; this is usually less than $30 \%$ due to imperfect overlap of the two wavelengths [38]. This was found to be $15.70 \%$. In contrast, the negative control (liposomes incorporating BSA-Atto680) was found to have a co-incidence value of $0.22 \pm 0.10 \%, n=3$ (Figure 4B). Biotin-tagged liposomes labeled with AF647-tagged streptavidin were found next to have a coincidence value of $9.20 \%$ (Supplementary Figure 3B). Finally, Wnt3A-Atto680-tagged DiO liposomes were found to have co-incidence values of $2.51 \pm 0.35 \%$, $\mathrm{n}=3$ (Figure 4C). BSA and Wnt3A-Atto680-tagged 
(A)
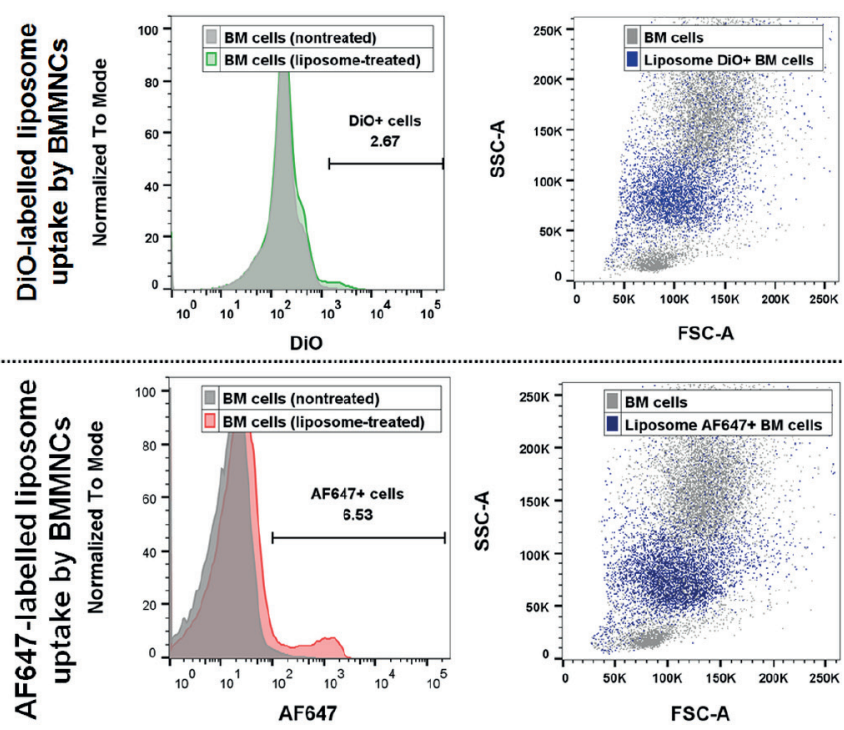

(C)
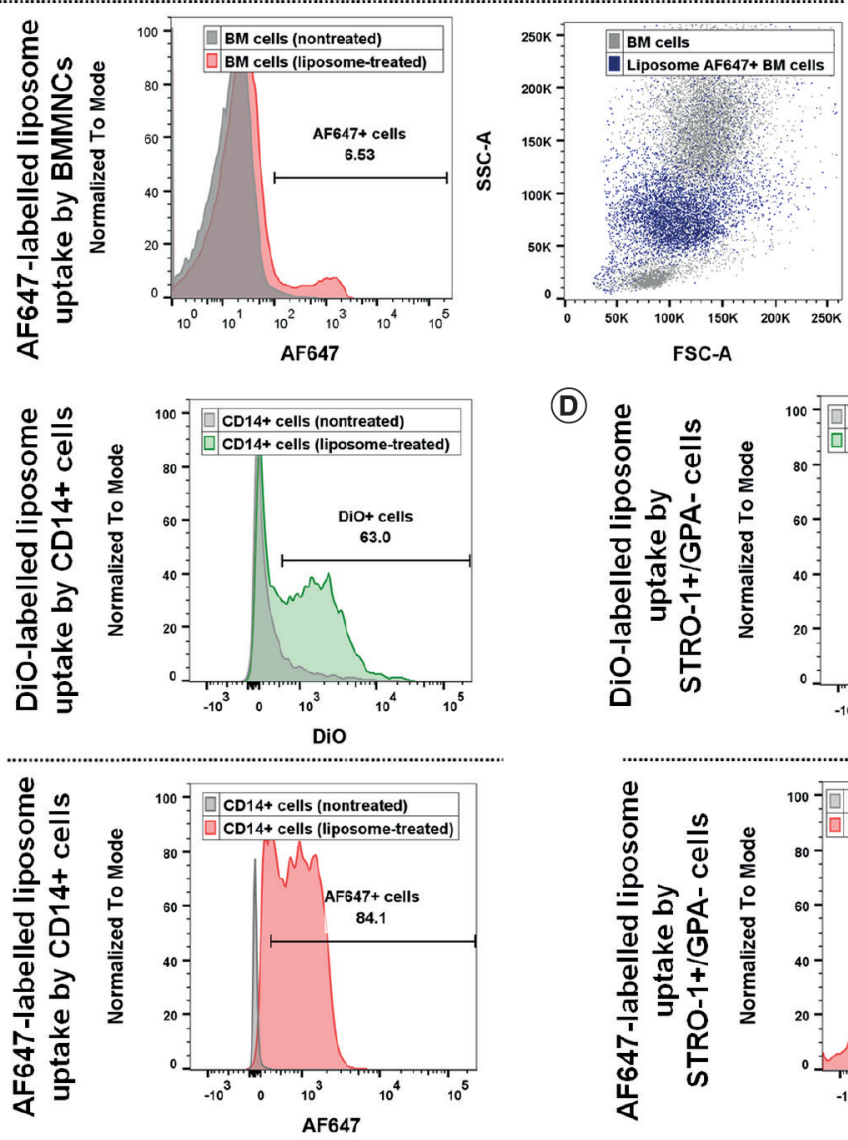

(D)
(B)

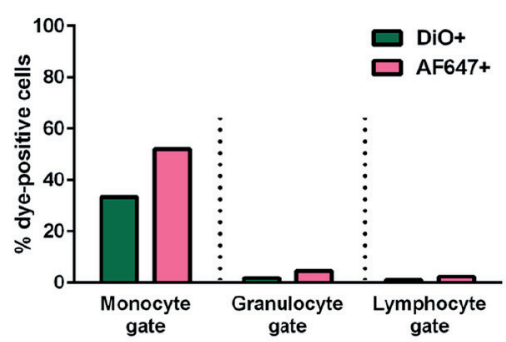

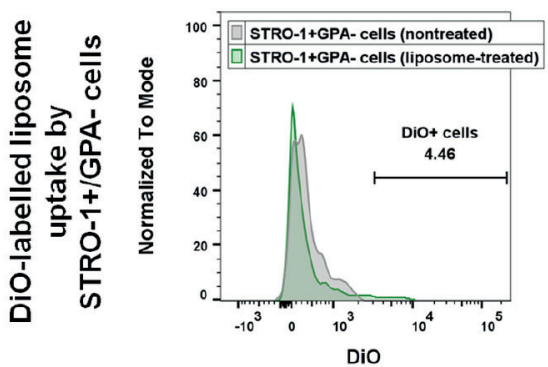

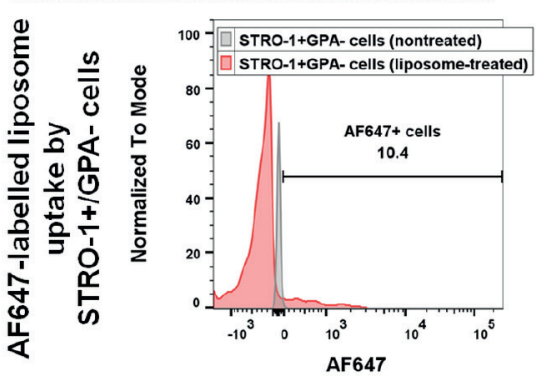

(E)
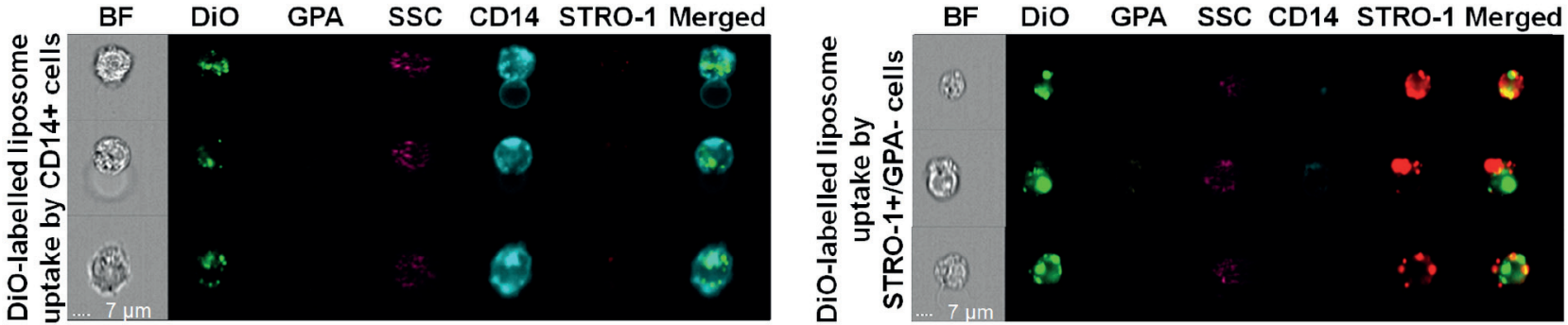

PEGylated DiO liposomes had comparable values of coincidence to the unPEGylated samples (values of 0.27 and 2.84\%, respectively; Supplementary Figure 3C).
Second, TIRFM was used to measure the colocalization of fluorescent tags. TIRFM is a technique that allows for the high-sensitivity detection of fluorophore- 
Figure 5. PEGylated DOPC liposomes are taken up by monocytes and skeletal progenitor cells within mixed populations of human bone marrow. (A) Liposomes were labeled by two different strategies (passive incorporation of DiO or covalent attachment of AF647) and human bone marrow populations were subsequently examined for liposome uptake/association by flow cytometry. Overall cell association was greater for AF647-tagged liposomes than for DiO (left panels, A) but in both cases predominant association was in cells in the 'monocyte' gate based on light scattering properties (right panels, [A]). (B) Quantification of DiO+ or AF647 cells within the cell populations gated for monocyte, granulocyte and lymphocyte demonstrated very low uptake in granulocyte- and lymphocyte-gated cells compared with monocyte-gated cells. Further analysis of populations within the monocyte scatter gate by staining for CD14, STRO-1 and GPA antibodies revealed a higher uptake in CD14+ monocytes (C) compared with STRO-1+/GPAosteoprogenitors (D). Intracellular localization of the particles was confirmed for the CD14+ monocytic cells (E left) as well as the STRO $-1^{+} / \mathrm{GPA}$ - skeletal stem cell containing fraction (E right) using imaging flow cytometry.

BF: Brightfield; BM: Bone marrow; DOPC: 1,2-dioleoyl-sn-glycero-3-phosphocholine; GPA: Glycophorin A; STRO-1: Stromal cell surface marker-1.

tagged molecules immobilized and spatiality isolated on a glass coverslip (see schematic presentation in Figure 4D). Labeled proteins and liposomes were incubated for a period of $5 \mathrm{~min}$ before imaging. The degree of colocalization of labeled Wnt3A protein with $\mathrm{DiO}$ labeled liposomes was significantly higher than in control-labeled BSA samples $(31.15 \pm 19.68 \%$ vs 6.89 $\pm 35.89 \%$ for BSA; $\mathrm{p}<0.01 ; \mathrm{n}=27$; Figure $4 \mathrm{E} \& \mathrm{~F}$ ) and was similar to the positive control, labeled streptavidin $33.71 \pm 25.28 \%(\mathrm{p}=0.6$; Supplementary Figure 3D $)$.

Together these imaging techniques demonstrate direct interaction of Wnt3A protein with liposomal carriers and that the stabilization of Wnt3A activity is likely to depend upon direct interaction of the protein with the synthetic lipid membrane.

Liposomes associate with monocyte \& stromal cell/stem cell fractions of human bone marrow cell populations

Circulating or locally delivered liposomal preparations are likely to encounter a mixed cellular milieu with only a proportion of therapeutic dose available to a given cell population. To determine the representative uptake in primary cell populations likely to be present in vivo at bone injury sites, liposome-cell association was measured in freshly isolated human BMMNCs by flow cytometry. DOPC-PEG liposomes were either labeled with $\mathrm{DiO}$ or with streptavidin-AF647 for tracking the distribution of lipid molecules following cell association.

After exposure of human BMMNCs to dye-labeled liposomes for $3 \mathrm{~h}$, a small proportion of the entire BMMNC fraction became dye-positive, indicating cellular liposome association (2.67 and $6.51 \%$ for $\mathrm{DiO}$ and $\mathrm{AF} 647$, respectively) (Figure 5A). In flow cytometry scatter plots of BMMNCs, cells of different populations/lineages (e.g., granulocytes, monocytes, lymphocytes) cluster based on their light-scattering properties (see Supplementary Figure 4A for details of gating of different BMMNC populations). The majority of this association was with cells that clustered with cells with similar light scattering properties to monocytes, but which also include skeletal/stromal stem/ progenitor populations. In this population $33.40 \%$ of cells were positive for $\mathrm{DiO}$ and $52.00 \%$ for AF647. In contrast, only $1.82 \%$ were positive for $\mathrm{DiO}$ and $4.56 \%$ positive for AF647 in the granulocyte gate, and 1.03\% positive for $\mathrm{DiO}$ and $2.35 \%$ positive for $\mathrm{AF} 647$ in the lymphocyte gate (Figure 5B).

We have previously shown that the 'monocyte' gated cell population includes stem and progenitor cells, which are the intended cellular targets of Wnt activation in bone marrow and at injury sites [25]. To determine the relative association of liposomes with these different cell populations we immunostained dyelabeled liposome-exposed cells for the cell surface antigens CD14, STRO-1 and GPA. Liposomes associated to a higher degree with $\mathrm{CD} 14^{+}$monocytes (63.0 and $84.5 \%$ for $\mathrm{DiO}$ - or AF647-labeled liposomes, respectively; Figure $5 \mathrm{C}$ ) than with $\mathrm{STRO}-1^{+} / \mathrm{GPA}^{-}$skeletal progenitors (4.5 vs $10.4 \%$ for $\mathrm{DiO}$ - or AF647-labeled liposomes, respectively; Figure 5D). In addition, we used Image Stream flow cytometry to determine whether DiO-labeled liposomes were internalized or remained adherent to the cell surface. In both $\mathrm{CD} 14^{+}$monocytes and $\mathrm{STRO}-1^{+} / \mathrm{GPA}^{-}$skeletal stem cells $\mathrm{DiO}$ was present as a punctate intracellular signal, indicated significant uptake within these cell populations (Figure 5E).

Together these data indicate that PEGylated liposomes associate with human bone marrow cells, primarily interacting with monocyte and stem/progenitor cells rather than granulocytes and lymphocytes.

The association of liposomes with bone marrow stromal cells is rapid \& is not negatively affected by PEGylation

To determine whether PEGylation affected the rate of association of liposomes with stromal stem or prgenitor cells, we exposed cultured, adherent BMSCs to dyelabeled liposomes and used FACS to quantify the time course of association.

Liposome association with cells was rapid, and within $3 \mathrm{~h}$ of incubation approximately $50 \%$ of cells stained positively (above a threshold set by the highest fluorescent intensity of an unstained sample; Supplementary Figure 4B), but became more gradual 
(A)

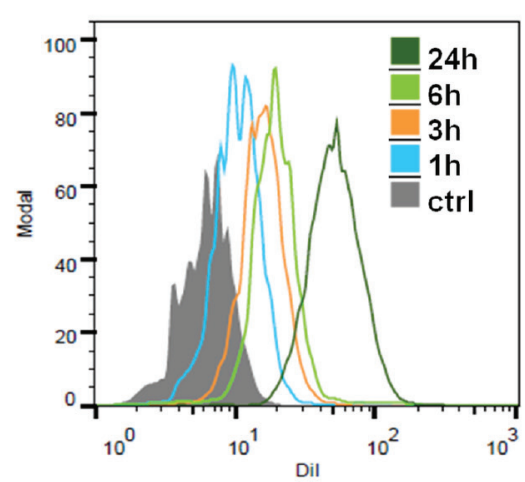

(C)

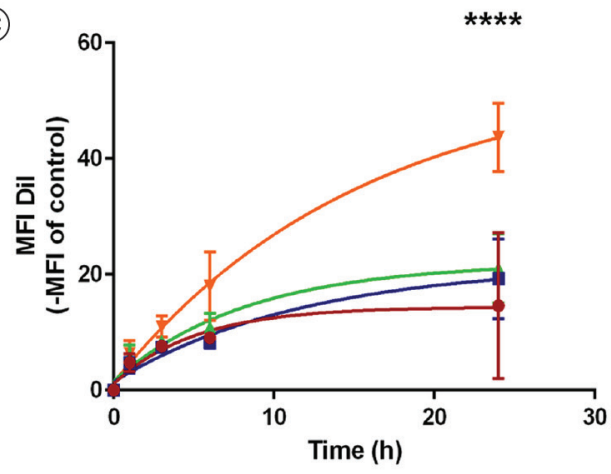

(E)
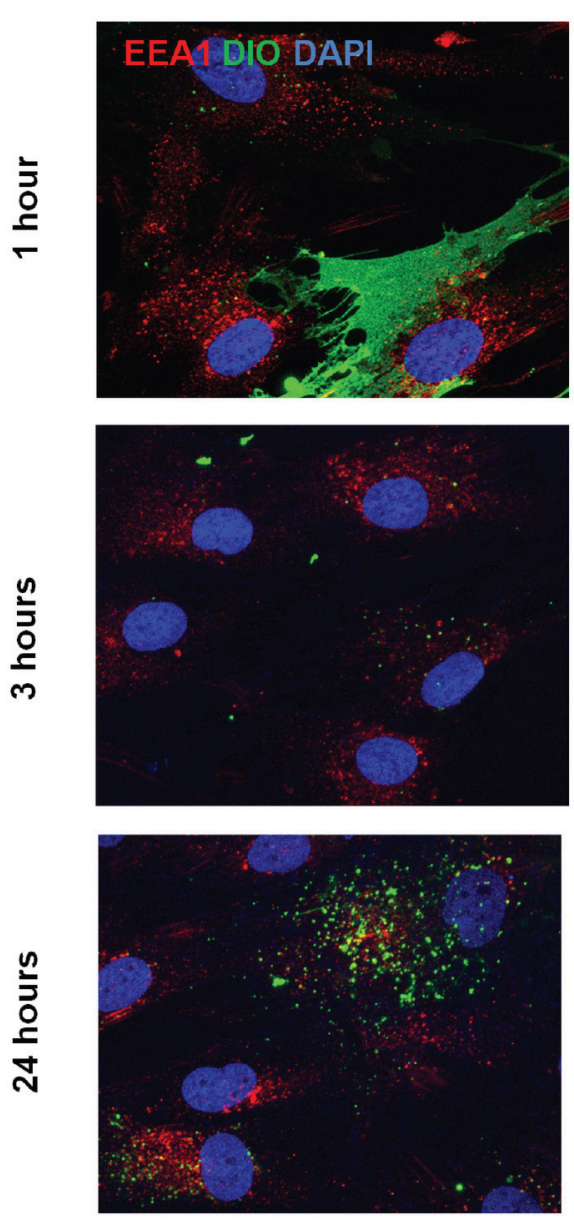

(B)

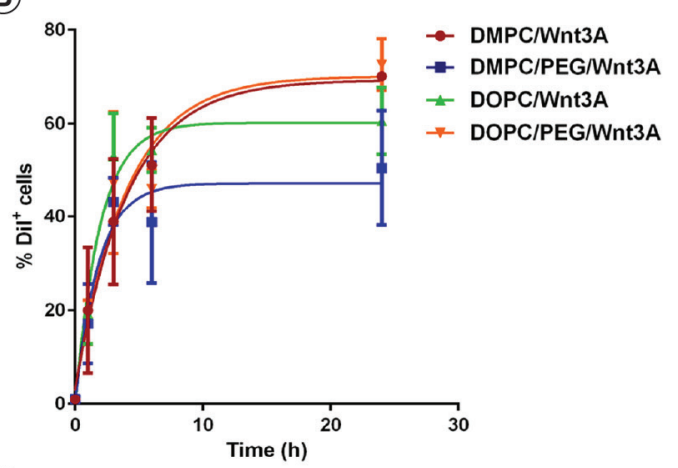

(D)
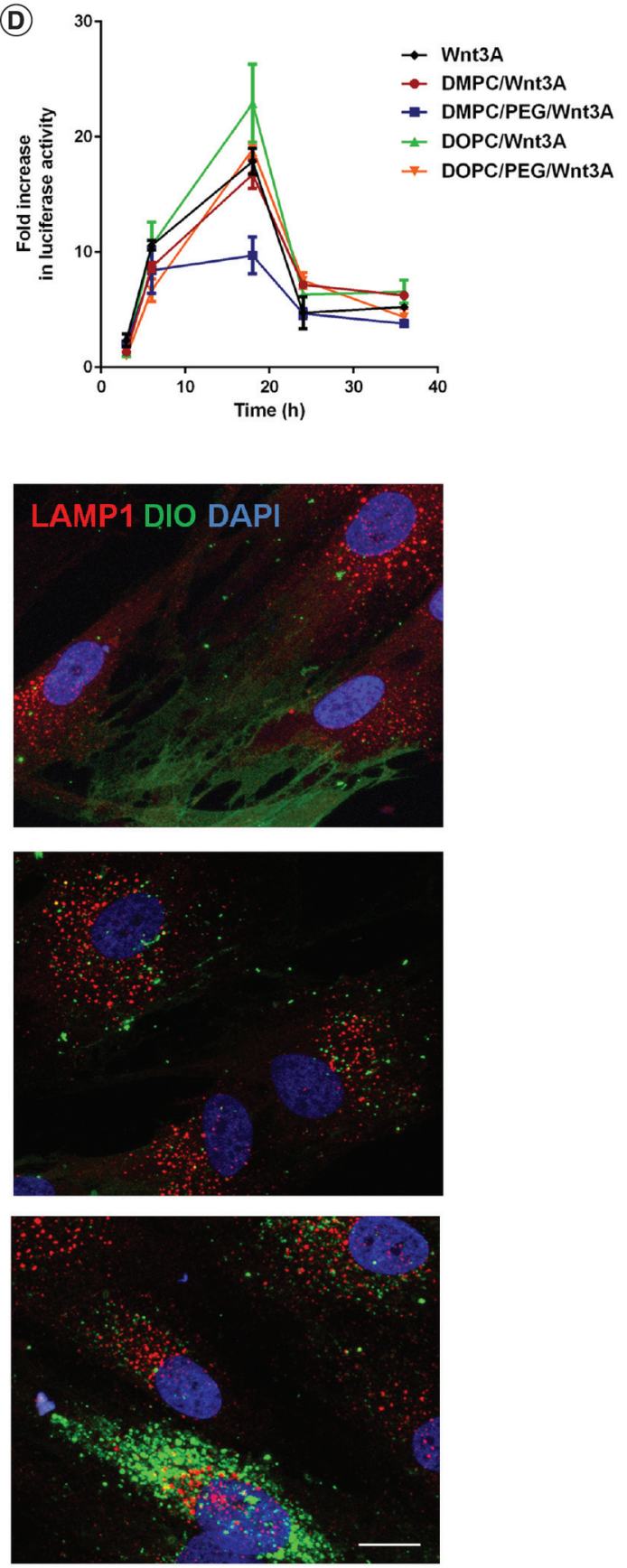
Figure 6. Liposome associate rapidly with stromal cells of the bone marrow. Flow cytometry analysis of the uptake Dil-labeled liposomes by adherent stromal cells of the bone marrow after 1-, 3-, 6- and 24-h incubation. An increase in cell fluorescence (A) was measured after liposome incubation, as presented on the histograms representing cells exposed to liposomes (colored) versus control nonexposed cells (gray). For quantification of cells that were positive for the liposomes, all cells gated as positive showed a fluorescence intensity greater than that detected on $99 \%$ of the cells not incubated with the liposomes. All liposome preparations were taken up rapidly by cells, resulting in $20 \%$ of the cells positively stained with Dil dye within an hour of incubation (B). ${ }^{*} p<$ 0.05 for DOPC/PEG liposomes versus DMPC/PEG liposomes. The MFI of the geometric mean for Dil is higher for DOPC/PEG versus all other preparations $\left(C_{;} * * * * p<0.0001\right)$. Data presented as mean $\pm S D, n=3$. MFI, mean fluorescence intensity. (D) Rate of activation of the Wnt signaling pathway in the reporter cell line was similar between all preparations. (E) Confocal micrographs representing intracellular localization of DiO-labeled liposomes (green) after 1, 3 and $24 \mathrm{~h}$ of incubation in stromal cells. Cells were costained with DAPI (blue), and the endosomal marker EEA1 (red) or the lysosomal marker LAMP1 (red). Magnification $63 \times$, scale bars $=20 \mu m$. Cell uptake was heterogeneous and the majority of the liposomes did not colocalize with the endocytic pathway vesicles. DAPI: 4',6-diamidino-2-phenylindole; DMPC: 1,2-dimyristoyl-sn-glycero-3-phosphocholine;

DOPC: 1,2-dioleoyl-sn-glycero-3-phosphocholine.

at later time-points, with a maximum of approximately $70 \%$ positively stained cells for the latest time-point measured, $24 \mathrm{~h}$ (Figure 6A \& B). The difference in association between various liposome compositions was not significant after 1,3 and $6 \mathrm{~h}$ of incubation, but association of DOPC/PEG liposomes labeled with DiI at $24 \mathrm{~h}$ measured by mean fluorescence intensity was significantly higher than other preparations (by a factor of 2.4; $p<0.0001$ ). The kinetics of association also differed, with a value for Kd of 18.33 for DOPC liposomes, compared with 3.35, 9.86 and 6.53 for DMPC, DMPC/PEG and DOPC, respectively (Figure 6C). We also found that PEGylated liposome preparations led to slightly decreased cell viability at 24 and $48 \mathrm{~h}$ in stromal cell populations (Supplementary Figure 5).

Despite subtle differences in the kinetics of association, we found that the rate of activation of the Wnt signaling pathway in the reporter cell line was similar between preparations, with no delay or advance in the activation of signaling (Figure 6D).

We next examined the association of liposomes with cells by confocal microscopy and tested the hypothesis that liposomes are endocytosed and partitioned to the endosomal/lysosomal pathway following cell interaction. Cultured BMSCs were exposed to DiOlabeled liposomes for 1, 3 or $24 \mathrm{~h}$, fixed, and imaged after staining with endosomal or lysosomal markers. The results showed increased intracellular localization of $\mathrm{DiO}$ liposomes with longer incubation periods (representative images of the time points shown in Figure $6 \mathrm{E}$ ). At the earlier time point, more cells presented with membrane staining, compared with later time points. Furthermore, there was little indication that liposomes colocalized with any of the stained cellular compartments (endosomes or lysosomes).

These data indicate that both PEGylated and unPEGylated liposomes rapidly associate with BMSCs, that they activate the Wnt signaling pathway to a similar degree and with similar kinetics to native protein, and that they initially partition to the membrane prior to uptake and intracellular partition.
PEGylated liposomes activate Wnt signaling \& expand osteoprogenitors in human bone marrow

Finally, to address whether Wnt3A PEGylated liposomes promote Wnt pathway activation to the same extent as Wnt3A alone, we measured $\beta$-catenin expression in MG63 osteoblastic cells after $1 \mathrm{~h}$ of incubation (Figure 7A). The expression of active, dephosphorylated $\beta$-catenin was increased to the same extent as after Wnt3A stimulation alone. Next, to assess whether Wnt3A PEGylated liposomes promote stem cell commitment in mixed populations of cells, we exposed freshly isolated human BMMNCs cells to either PEGylated liposome associated or unassociated Wnt3A protein. Following a 24-h stimulation, the expression of AXIN2, a Wnt target gene, increased by a factor of 2.40 $\pm 0.36(\mathrm{p}<0.01)$ in comparison to nonstimulated control, similar to that of Wnt3A alone control (Figure 7B). We also measured an increase (by a factor of $1.19 \pm$ 0.07; $<<0.05$ ) in the frequency of STRO- ${ }^{\text {bright }} / \mathrm{GPA}^{-}$ fraction of BM cells, containing putative skeletal stem cells and osteoprogenitors, again similar to the effect exerted by Wnt3A alone (Figure 7C). We then cultured BMMNCs transiently stimulated with Wnt liposomes as adherent stromal cell cultures (BMSCs) for 14 days. In two out of three donors, transient Wnt stimulation increased ALPase activity at day 14, with no significant difference between the liposomal-Wnt3A versus unincorporated Wnt3A-treated cells (Figure 7D).

These experiments confirmed that the protein in the PEGylated liposomal formulation mimics the actions of the unincorporated protein, and suggests that PEGylated liposome preparations are suitable vehicles for delivery of active Wnt3A protein in mixed cellular milieu.

\section{Discussion}

Stable, circulating carriers such as liposomal or polymer nanoparticles have significant potential for delivery of hydrophobic proteins and molecules for treatment of bone disease and injury. Wnt-carrying liposomes have 
(A)

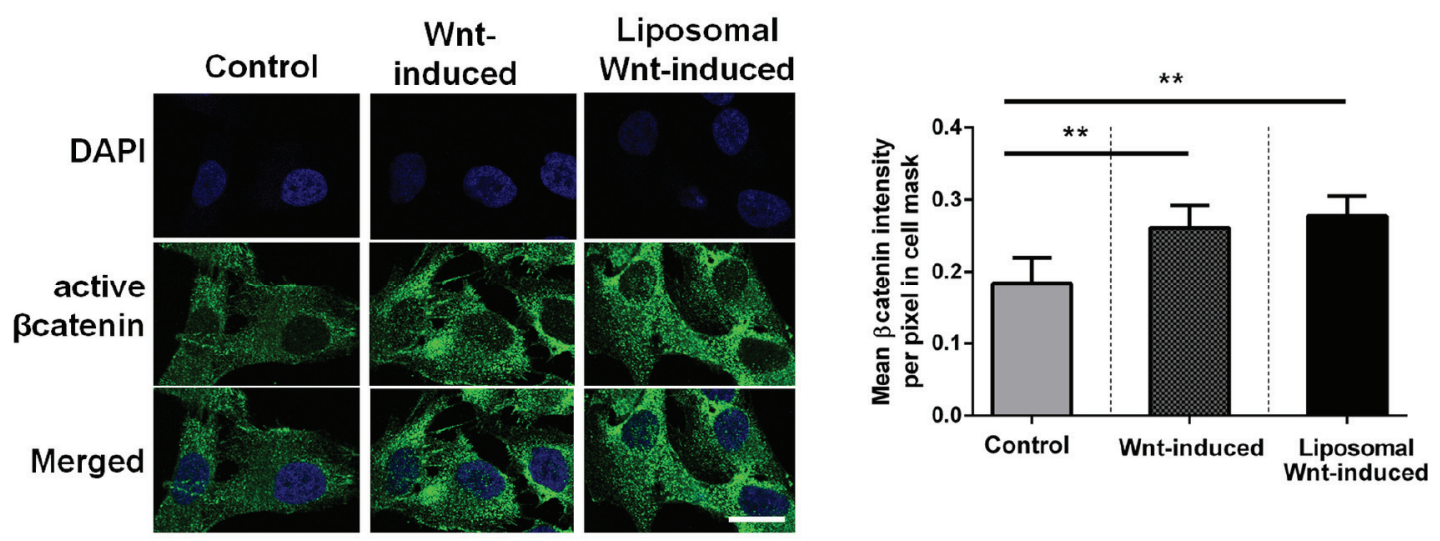

(B)

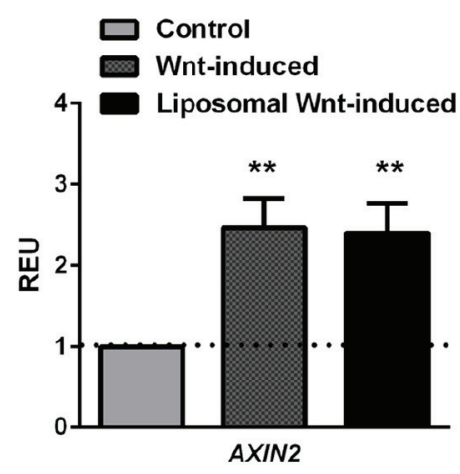

(C)

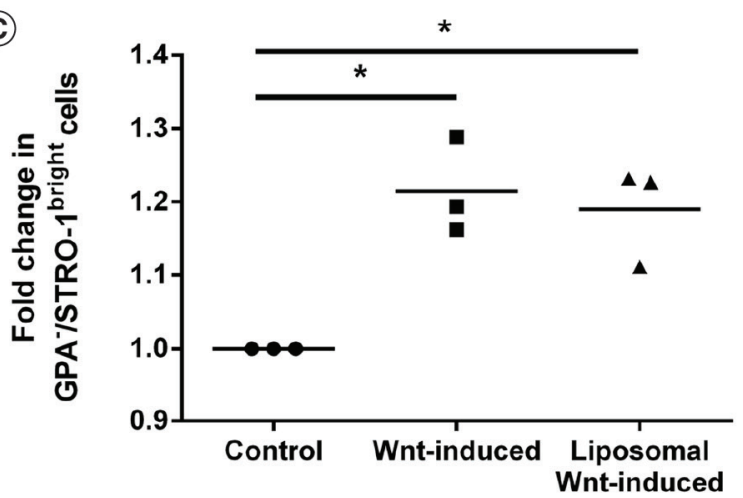

(D)

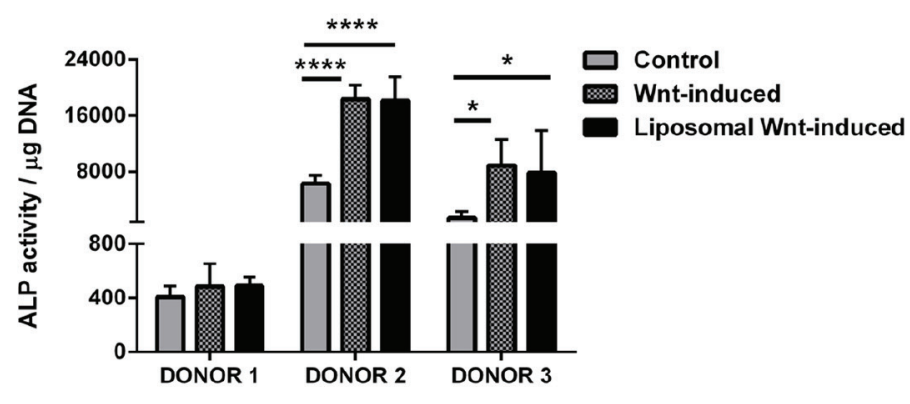

Figure 7. PEGylated liposomes activate Wnt signaling and affect osteoprogenitors in human bone marrow. PEGylated Wnt3A DOPC liposomes caused an increase in active $\beta$-catenin expression, versus control samples containing liposomes without Wnt3A, comparable to that of Wnt3A lone (A). These liposomes also increased Wnt target gene expression, AXIN2 (B), in STRO-1-enriched BM cells over $24 \mathrm{~h}$, as well as increased the STRO-1 bright/GPA- putative skeletal progenitor population (C). After 14 days of culture, cells transiently exposed to liposomal Wnt presented with increased bone marker expression, ALP (D).

ALP: Alkaline phosphatase; BM: Bone marrow; GPA: Glycophorin A.

been shown to have potential as local delivery agents for bone repair [29], but may have limited utility as circulating carriers due their rapid opsonization and clearance. By directly comparing the incorporation of Wnt3A protein at different stages of the fabrica- tion of liposomes, we found that PEGylated DMPC and DOPC liposomes can retain high Wnt signaling activity, but only when the protein was added after extrusion. Single molecule spectroscopy techniques allowed us to show direct interaction between Wnt3A 
molecules and liposomes, and active liposome preparations associated with and activated the Wnt signaling pathway in target cells with similar kinetics to native protein. These data illustrate the utility of PEGylated Wnt3A liposomes as circulating activators of Wnt signaling.

In previous work, it was found that only DMPC was able to sustain Wnt3A-liposome activity, and that inclusion of PEGylated lipids completely abrogated it [30]. Other lipids, such as 1,2-dipalmitoyl-sn-glycero3-phosphocholine (DPPC), 1-myristoyl-2-palmitoylsn-glycero-3-phosphocholine (MPPC) and 1-palmitoyl-2-oleoyl-sn-glycero-3-phosphocholine (POPC), or combinations of them, with or without PEGylated DSPE, were unable to support Wnt activity. In contrast, in our experiments, we found that activity of Wnt3A in both unPEGylated and PEGylated DOPC liposomes was preserved, and that PEGylation of DMPC liposomes reduced activity by only approximately $50 \%$. This was dependent on the addition of Wnt post extrusion, and was time dependent, with increases in activity upon incubation for up to $24 \mathrm{~h}$, as has been shown for DMPC liposomes previously [28]. There are several possible reasons why certain lipids may inhibit Wnt activity - these include low incorporation of protein in liposomes during fabrication; denaturation during the incorporation process; incorrect presentation of protein to recipient cells; or insufficient interaction/ binding of liposomes to target cell populations.

It is unlikely that differences in incorporation efficiency are responsible for the differences we measured in activity - similar amounts of protein were measured in DMPC compared with PEGylated DMPC liposomes, despite the latter being less active in cell culture assays than the former. This conclusion is supported by previous work where high incorporation of Wnt3A was observed in inactive MPPC, DPPC and POPC preparations [30]. In contrast, DOPC liposomes contained similar amounts of protein and had similar activities regardless of PEGylation. Therefore, PEGylation does not affect protein incorporation or activity per se, but instead reduces the ability of liposomes of certain chemistries to activate signaling. In parallel, we found similar cellular liposome association with cells for all preparations (Figure 6; except for DOPC liposomes, which had a significantly higher association), regardless of activity. For this reason, it is also unlikely that differences in the degree of liposome-cell interaction are responsible for differences in activity.

Instead, we consider it probable that subtle differences in liposomal chemistry may modulate the mechanism of Wnt3A protein presentation to Frizzled receptors at the cell surface. Both DMPC and DOPC lipids are in a liquid crystalline form at body tempera- ture (which we and other have found to be necessary for Wnt association with liposomes [39]), but DMPC and DOPC differ in their size and saturation - DMPC has a saturated 14 carbon acyl tail, while DOPC is longer (18 carbons) and is monounsaturated. Wnt3A is modified with palmitoleic acid at Ser209 and palmitate at Cys77 [40] (although the relevance of the latter modification in vivo has recently been disputed [41]). It has been suggested that one or both of these modifications facilitate stable anchorage of the protein in the synthetic lipid bilayer [30], although hydrophobic regions of the protein may also be responsible. Due to the presence of a single double bond, unsaturated phospholipids such as DOPC have 'kinked' acyl chains, and do not align in the same manner as saturated phospholipids such as DMPC. This leads to looser packing of phospholipids in DOPC compared with DMPC liposomes, which may facilitate higher protein mobility, less stable protein/membrane binding and an improved ability for membrane-associated proteins to undergo conformational change $[42,43]$. To date, the only data available on the nature of the interaction of Wnt proteins with Frizzled receptors comes from Xenopus XWnt8, where the palmitoleic acid residue at Ser187 (equivalent to the same modification at Ser209 on mammalian Wnt3A) is involved in the direct interaction with Frizzled [27]. Therefore, if the palmitoleic acid modification on Wnt3A is involved in liposome anchorage but is also necessary for Frizzled interaction, there must be an active (energetically unfavorable) process by which the Wnt $3 \mathrm{~A}$ is reoriented at the cell surface to promote Frizzled engagement. Regardless of the putative mechanism of Wnt3A binding to liposomes, significant changes in protein orientation/ conformation of this nature are likely to be promoted by more loosely packed liposome lipids such as DOPC as compared with DMPC. This may be particularly true when PEG groups are present at the liposomal surface, which may inhibit close liposome-cell interaction. Future studies may address how phospholipid composition (for example, by varying the proportion of synthetic lipids and/or cholesterol) affects the stability of Wnt3A protein interaction with liposomes using biochemical and biophysical techniques.

Using TCCD and TIRFM, we confirmed direct interaction of labeled Wnt3A protein with liposome carriers. TCCD has previously been used for investigation of protein aggregation $[35,44]$ or for direct visualization of protein interactions on cell membranes [45], but to our knowledge, this is for the first time it has been employed to study protein/liposome interactions. A similar technique, dual-color fluorescence-burst analysis (DCFBA) has been developed to measure the leakage of entrapped fluorescent molecules from lipo- 
somes [46], but TCCD in conditions of fast flow offers a number of advantages. These include a lack of reliance on the relatively slow diffusion of liposomes $(\sim 3$ $\mu \mathrm{m}^{2} \mathrm{~s}^{-1}$ compared with $>50 \mu \mathrm{m}^{2} \mathrm{~s}^{-1}$ for proteins) which limits the data that can be collected in a static confocal spot, and no requirement for a binding partner (which may disrupt the membrane), instead detecting whether or not the species interacts with the liposomes. TCCD also offers a number of potential advantages for further elucidating the nature and degree of interaction of labeled fluorescent proteins with liposomes. For example, the sensitivity of TCCD allows one to determine not only if two fluorophores are coincident, but also how many are present, measurements important prior to use of drug-containing liposomes as pharmaceutical agents. Lifetime and Förster resonance energy transfer measurements, in combination with steady-state TCCD and TIRFM measurements may be useful in determining the precise biophysical association of the protein with the liposomal membrane or with other carriers [47]. While anchorage of one of Wnt3A's lipid adducts into a membrane is an attractive mechanism for its association with synthetic membranes such as liposomes, an alternate hypothesis is that hydrophobic domains in the protein may facilitate this interaction. Lifetime measurements of a protein-attached fluorophore may help resolve this question, as fluorescence lifetime is very sensitive to solvent polarity and thus could be used to infer whether a portion of the protein is membrane embedded. Further single-molecule studies of such interactions will provide fundamental insight into the biophysical interactions involved in these transport mechanisms.

Wnt3A-associated PEGylated DOPC liposomes rapidly associated with human bone marrow mononuclear cells extracted from patients following hip arthroplasty. As might be expected, the highest uptake was in the $\mathrm{CD} 14^{+}$monocyte population, which include macrophages at various stages of cell commitment. Macrophages are the principle cell type responsible for clearance of circulating liposomes, a process that has been exploited for many years to selectively deplete macrophages using liposome-encapsulated clodronate [48]. However, there was also significant uptake by relatively rare STRO- $1^{+} / \mathrm{GPA}^{-}$cells, a putative skeletal stem cell population that gives rise to bone tissue. We have previously shown that temporally controlled Wnt stimulation of these cells promotes an increase in the frequency of STRO-1+/GPA- cells in human bone marrow, and a subsequent increase in osteogenesis [25]. In similar experiments reported here, we find an effect of Wnt-associated liposomes not significantly different to native Wnt3A protein. Interestingly, the kinetics of activation of Wnt signaling were very similar, regardless of whether Wnt3A was delivered in its native form or incorporated in liposomes, which supports the notion that cell-surface association of liposome-associated Wnt3A is sufficient to induce activation. Despite this, liposomes were taken up and internalized by primary human cells, but we did not address whether liposome uptake was necessary for activation. Future studies may seek to address this by the use of specific inhibitors of endocytic pathways, with the caveat that receptor internalization is necessary for Wnt signaling (and so it may be difficult to separate the effects of liposome uptake vs receptor complex internalization).

It is worth noting that nanocarriers such as liposomes can be targeted to different cells or tissues by the inclusion of motifs that are recognized or adhere to markers in certain tissues. This has recently been exploited to localize nanoparticles to sites of bone damage or repair [49,50]. Our experiments showing PEGylated Wnt3A-carrying nanoparticles are stable and induce activity in clinically relevant human cell isolates now provides the opportunity for spatiotemporal targeting of Wnt3A protein for anabolic therapies in medicine.

\section{Conclusion}

In summary, we have demonstrated that a number of 'stealth' liposomal formulations retain Wnt activity in vitro and may be used in the future as delivery agents to stromal/stem cell populations within the bone marrow niche. These results give further encouragement that a liposomal Wnt formulation could be used in future studies in systemic administration for promoting bone anabolism or for controlled delivery to fractures for repair in patients. This will be the focus of ongoing work.

\section{Supplementary data}

To view the supplementary data that accompany this paper please visit the journal website at: www.futuremedicine.com/ doi/full/10.2217/nnm-2016-0386

\section{Author contributions}

AA Janeczek contributed in conception and design, collection and/or assembly of data, data analysis and interpretation, manuscript writing and final approval of manuscript. E Scarpa contributed in collection and/or assembly of data, data analysis and interpretation, and final approval of manuscript. MH Horrocks contributed in collection and/or assembly of data, data analysis and interpretation, and final approval of manuscript. RS Tare contributed in conception and design, data analysis and interpretation, and final approval of manuscript. CA Rowland contributed in data analysis and interpretation, and final approval of manuscript. D Jenner contributed in collection and/or assembly of data, data analysis and interpretation, and final approval of manuscript. TA Newman 
contributed in conception and design, data analysis and interpretation, and final approval of manuscript. SF Lee contributed in conception and design, data analysis and interpretation, and final approval of manuscript. ROC Oreffo contributed in conception and design, data analysis and interpretation, financial support and final approval of manuscript. ND Evans contributed in conception and design, financial support, data analysis and interpretation, manuscript writing, and final approval of manuscript.

\section{Acknowledgements}

The authors thank A West for help with gas chromatography, and $\mathrm{R}$ Smith and $\mathrm{U}$ Jonnalagadda for help with image analysis after $\beta$-catenin staining. The authors thank the orthopedic surgeons at Southampton General Hospital for provision of bone marrow samples.

Financial \& competing interests disclosure

The authors acknowledge funding support from the Medical Research Council, UK (grant number MR/J004103/1), Wessex
Medical Research (grant number SO2), UoS Research Management Committee and the Institute for Life Sciences, Southampton. The authors would like to thank the Royal Society for the University Research Fellowship of Steven F Lee (UF120277). The funding bodies had no involvement in study design, collection, analysis and interpretation of data, writing of the report and in the decision to submit the article for publication. The authors have no other relevant affiliations or financial involvement with any organization or entity with a financial interest in or financial conflict with the subject matter or materials discussed in the manuscript apart from those disclosed.

No writing assistance was utilized in the production of this manuscript.

\section{Open access}

This article is distributed under the terms of the Creative Commons Attribution License 4.0 which permits any use, distribution, and reproduction in any medium, provided the original author(s) and the source are credited. To view a copy of the license, visit http://creativecommons.org/licenses/by/4.0/

\section{Summary points}

PEGylated liposomes can be fabricated with uniform physical properties, \& are stable for prolonged time periods

- PEGylated liposomes have a size distribution of approimately $100 \mathrm{~nm}$ and are stable for at least 21 days.

Wnt3A protein is active in 'stealth' liposomal preparations

- Activity of Wnt3A-containing liposomes is dependent on the method of addition.

- Incorporation with the protein prior to extrusion resulted only in active unPEGylated DMPC liposomes. In contrast, incubation of liposomes with Wnt3A post extrusion resulted in active DMPC, DOPC and PEGylated DOPC liposomes. Again, DMPC liposomes were not active.

- This was not due to a lack of protein incorporation, as the amount of Wnt3A protein present in the lipid pellets did not correlate with activity.

Wnt3A association with liposomes can be visualized at the single-molecule level

- Two color-coincidence detection and TIRF are techniques that allow visualization of direct interaction of labeled proteins with liposomes.

- For the first time, we show that Wnt3A directly associates with unPEGylated and PEGylated liposomes.

Liposomes associate with monocyte \& stromal cell/stem cell fractions of human bone marrow cell populations

- After exposure of human bone marrow mononuclear isolates, PEGylated liposomes predominantly associate with CD14+ monocytes, but also with STRO-1+/GPA- skeletal stem cells.

- Liposomes do not associated significantly with granulocytes or lymphocytes.

The association of liposomes with bone marrow stromal cells is rapid \& is not negatively affected by PEGylation

- PEGylation of DOPC liposomes does not affect the number of cells that associate with them, but increases the mean degree of uptake over $24 \mathrm{~h}$.

PEGylated Wnt liposomes activate Wnt signaling \& expand osteoprogenitors in human bone marrow

- Exposure of STRO-1+ bone marrow stromal cells increases nuclear localization of $\beta$-catenin and AXIN2 expression, indicating activation of Wnt signaling.

- PEGylated DOPC Wnt3A liposomes expand osteoprogenitors and induce osteogenic differentiation to a similar degree to native protein.

Conclusion

- Active Wnt protein-containing PEGylated liposomes may have utility for systemic administration for bone repair.

\section{References}

1 Lasic DD. Liposomes: From Physics to Applications.ISBN-10: 0444895485. Elsevier, London, UK (1993).
2 Walsh TJ, Finberg RW, Arndt C et al. Liposomal amphotericin $\mathrm{B}$ for empirical therapy in patients with persistent fever and neutropenia. N. Engl. J. Med. 340(10), 764-771 (1999). 
3 Glück R, Mischler R, Brantschen S, Just M, Althaus B, Cryz SJ. Immunopotentiating reconstituted influenza virus virosome vaccine delivery system for immunization against hepatitis A. J. Clin. Invest. 90(6), 2491-2495 (1992).

4 Barenholz Y(Chezy). Doxil ${ }^{\circledR}$ - the first FDA-approved nanodrug: lessons learned. J. Control. Release 160(2), 117-134 (2012).

5 Pattni BS, Chupin VV, Torchilin VP. New developments in liposomal drug delivery. Chem. Rev. 115(19), 10938-10966 (2015).

6 Immordino ML, Dosio F, Cattel L. Stealth liposomes: review of the basic science, rationale, and clinical applications, existing and potential. Int. J. Nanomedicine 1(3), 297-315 (2006).

7 Maeda H, Wu J, Sawa T, Matsumura Y, Hori K. Tumor vascular permeability and the EPR effect in macromolecular therapeutics: a review. J. Control. Release 65(1), 271-284 (2000).

8 Petersen GH, Alzghari SK, Chee W, Sankari SS, La-Beck NM. Meta-analysis of clinical and preclinical studies comparing the anticancer efficacy of liposomal versus conventional non-liposomal doxorubicin. J. Control. Release 232, 255-264 (2016).

9 Monteiro N, Martins A, Reis RL et al. Liposomes in tissue engineering and regenerative medicine. J. R. Soc. Interface 11(101), 20140459 (2014).

10 Mickova A, Buzgo M, Benada O et al. Core/shell nanofibers with embedded liposomes as a drug delivery system. Biomacromolecules 13(4), 952-962 (2012).

11 Bonanomi MH, Velvart M, Weder HG. Fate of different kinds of liposomes containing dexamethasone palmitate after intra-articular injection into rabbit joints. J. Microencapsul. 4(3), 189-200 (1987).

12 Shmeeda H, Amitay Y, Tzemach D, Gorin J, Gabizon A. Liposome encapsulation of zoledronic acid results in major changes in tissue distribution and increase in toxicity. J. Control. Release 167(3), 265-275 (2013).

13 Gutman D, Golomb G. Liposomal alendronate for the treatment of restenosis. J. Control. Release 161(2), 619-627 (2012).

14 Zhang G, Guo B, Wu H et al. A delivery system targeting bone formation surfaces to facilitate RNAi-based anabolic therapy. Nat. Med. 18(2), 307-314 (2012).

15 Hengst V, Oussoren C, Kissel T, Storm G. Bone targeting potential of bisphosphonate-targeted liposomes: Preparation, characterization and hydroxyapatite binding in vitro. Int. J. Pharm. 331(2), 224-227 (2007).

16 Cho H, Stuart JM, Magid R et al. Theranostic immunoliposomes for osteoarthritis. Nanomedicine 10(3), 619-627 (2014).

17 Mann AP, Tanaka T, Somasunderam A, Liu X, Gorenstein DG, Ferrari M. E-selectin-targeted porous silicon particle for nanoparticle delivery to the bone marrow. $A d v$. Mater. 23(36), H278-H282 (2011).

18 Balemans W, Ebeling M, Patel N et al. Increased bone density in sclerosteosis is due to the deficiency of a novel secreted protein (SOST). Hum. Mol. Genet. 10(5), 537-543 (2001).
19 Gong Y, Slee RB, Fukai N et al. LDL receptor-related protein 5 (LRP5) affects bone accrual and eye development. Cell 107(4), 513-523 (2001).

20 Iyer SP, Beck JT, Stewart AK et al. A Phase IB multicentre dose-determination study of BHQ880 in combination with anti-myeloma therapy and zoledronic acid in patients with relapsed or refractory multiple myeloma and prior skeletal-related events. Br. J. Haematol. 167(3), 366-375 (2014).

21 Padhi D, Jang G, Stouch B, Fang L, Posvar E. Single-dose, placebo-controlled, randomized study of AMG 785, a sclerostin monoclonal antibody. J. Bone Miner. Res. 26(1), 19-26 (2011).

22 McClung MR, Grauer A, Boonen S et al. Romosozumab in postmenopausal women with low bone mineral density. $N$. Engl. J. Med. 370(5), 412-20 (2014).

23 Padhi D, Allison M, Kivitz AJ et al. Multiple doses of sclerostin antibody romosozumab in healthy men and postmenopausal women with low bone mass: a randomized, double-blind, placebo-controlled study. J. Clin. Pharmacol. 54(2), 168-178 (2014).

24 Keizer RJ, Huitema ADR, Schellens JHM, Beijnen JH. Clinical pharmacokinetics of therapeutic monoclonal antibodies. Clin. Pharmacokinet. 49(8), 493-507 (2010).

25 Janeczek AA, Tare RS, Scarpa E et al. Transient canonical wnt stimulation enriches human bone marrow mononuclear cell isolates for osteoprogenitors. Stem Cells 34(2), 418-430 (2015).

26 Willert K, Brown JD, Danenberg E et al. Wnt proteins are lipid-modified and can act as stem cell growth factors. Nature 423(6938), 448-452 (2003).

27 Janda CY, Waghray D, Levin AM, Thomas C, Garcia KC. Structural basis of Wnt recognition by Frizzled. Science 337(6090), 59-64 (2012).

28 Dhamdhere GR, Fang MY, Jiang J et al. Drugging a stem cell compartment using Wnt3a protein as a therapeutic. PLoS ONE 9(1), e83650 (2014).

29 Minear S, Leucht P, Jiang J et al. Wnt proteins promote bone regeneration. Sci. Transl. Med. 2(29), 29ra30 (2010).

30 Morrell NT, Leucht P, Zhao L et al. Liposomal packaging generates Wnt protein with in vivo biological activity. PLoS ONE 3(8), e2930 (2008).

31 Popelut A, Rooker SM, Leucht P, Medio M, Brunski JB, Helms JA. The acceleration of implant osseointegration by liposomal Wnt3a. Biomaterials 31(35), 9173-9181 (2010).

32 Whyte JL, Smith AA, Liu B et al. Augmenting endogenous Wnt signaling improves skin wound healing. PLoS ONE 8(10), e76883 (2013).

33 Janeczek AA, Tare RS, Scarpa E et al. Transient canonical Wnt stimulation enriches human bone marrow mononuclear cell isolates for osteoprogenitors. Stem Cells 34(2), 418-430 (2016).

34 Chang VT, Fernandes RA, Ganzinger KA et al. Initiation of T cell signaling by CD45 segregation at "close contacts.". Nat. Immunol. 17(5), 574-582 (2016).

35 Horrocks MH, Tosatto L, Dear AJ et al. Fast flow microfluidics and single-molecule fluorescence for the 
rapid characterization of $\alpha$-synuclein oligomers. Anal. Chem. 87(17), 8818-8826 (2015).

Horrocks MH, Li H, Shim J-U et al. Single molecule fluorescence under conditions of fast flow. Anal. Chem. 84(1), 179-185 (2012).

37 Li H, Ying L, Green JJ, Balasubramanian S, Klenerman D. Ultrasensitive coincidence fluorescence detection of single DNA molecules. Anal. Chem. 75(7), 1664-1670 (2003).

38 Li H, Zhou D, Browne H, Balasubramanian S, Klenerman D. Molecule by molecule direct and quantitative counting of antibody-protein complexes in solution. Anal. Chem. 76(15), 4446-4451 (2004)

39 Jing W, Smith AA, Liu B et al. Reengineering autologous bone grafts with the stem cell activator WNT3A. Biomaterials 47, 29-40 (2015).

40 Takada R, Satomi Y, Kurata T et al. Monounsaturated fatty acid modification of Wnt protein: its role in Wnt secretion. Dev. Cell 11(6), 791-801 (2006).

41 Gao X, Hannoush RN. Single-cell imaging of Wnt palmitoylation by the acyltransferase porcupine. Nat. Chem . Biol. 10(1), 61-68 (2013).

42 Vanni S, Hirose H, Barelli H, Antonny B, Gautier R. A sub-nanometre view of how membrane curvature and composition modulate lipid packing and protein recruitment. Nat. Commun. 5, 4916 (2014).

43 Lindblom G, Orädd G. Lipid lateral diffusion and membrane heterogeneity. Biochim. Biophys. Acta 1788(1), 234-244 (2009).
44 Tosatto L, Horrocks MH, Dear AJ et al. Single-molecule FRET studies on alpha-synuclein oligomerization of Parkinson's disease genetically related mutants. Sci. Rep. 5 , 16696 (2015).

45 James JR, White SS, Clarke RW et al. Single-molecule level analysis of the subunit composition of the T cell receptor on live T cells. Proc. Natl Acad. Sci. USA 104(45), 17662-17667 (2007).

46 van den Bogaart G, Kusters I, Velásquez J, Mika JT, Krasnikov V, Driessen AJM. Dual-color fluorescenceburst analysis to study pore formation and protein-protein interactions. Methods 46(2), 123-130 (2008).

Bowen ME, Weninger K, Brunger AT et al. Single molecule observation of liposome-bilayer fusion thermally induced by soluble $\mathrm{N}$-ethyl maleimide sensitive-factor attachment protein receptors (SNAREs). Biophys. J. 87(5), 3569-3584 (2004).

48 Rooijen N Van, Sanders A. Liposome mediated depletion of macrophages: mechanism of action, preparation of liposomes and applications. J. Immunol. Methods 174(1-2), 83-93 (1994).

49 Swami A, Reagan MR, Basto P et al. Engineered nanomedicine for myeloma and bone microenvironment targeting. Proc. Natl Acad. Sci. USA 111(28), 10287-10292 (2014).

50 Jia Z, Zhang Y, Chen YH et al. Simvastatin prodrug micelles target fracture and improve healing. J. Control. Release 200, 23-34 (2015). 